\title{
ACTIVE-SET REDUCED-SPACE METHODS WITH NONLINEAR ELIMINATION FOR TWO-PHASE FLOW PROBLEMS IN POROUS MEDIA*
}

\author{
HAIJIAN YANG ${ }^{\dagger}$, CHAO YANG ${ }^{\ddagger}$, AND SHUYU SUN $\S$
}

\begin{abstract}
Fully implicit methods are drawing more attention in scientific and engineering applications due to the allowance of large time steps in extreme-scale simulations. When using a fully implicit method to solve two-phase flow problems in porous media, one major challenge is the solution of the resultant nonlinear system at each time step. To solve such nonlinear systems, traditional nonlinear iterative methods, such as the class of the Newton methods, often fail to achieve the desired convergent rate due to the high nonlinearity of the system and/or the violation of the boundedness requirement of the saturation. In the paper, we reformulate the two-phase model as a variational inequality that naturally ensures the physical feasibility of the saturation variable. The variational inequality is then solved by an active-set reduced-space method with a nonlinear elimination preconditioner to remove the high nonlinear components that often causes the failure of the nonlinear iteration for convergence. To validate the effectiveness of the proposed method, we compare it with the classical implicit pressure-explicit saturation method for two-phase flow problems with strong heterogeneity. The numerical results show that our nonlinear solver overcomes the often severe limits on the time step associated with existing methods, results in superior convergence performance, and achieves reduction in the total computing time by more than one order of magnitude.
\end{abstract}

Key words. two-phase flow, variational inequality, active-set reduced-space methods, nonlinear preconditioners, nonlinear elimination, parallel computing

AMS subject classifications. $76 \mathrm{~S} 05,68 \mathrm{~W} 10$

DOI. $10.1137 / 15 \mathrm{M} 1041882$

1. Introduction. Modeling and simulation of two-phase flow in porous media have been a major effort in reservoir engineering [37]. In addition to oil reservoir management, understanding and modeling of two-phase flow are crucial also to many environment issues. For example, one of the most attractive and practical solutions to reducing the $\mathrm{CO}_{2}$ emission problem is to inject and store $\mathrm{CO}_{2}$ in subsurface geological formations [43], such as depleted reservoirs and deep saline aquifers. The large capacity of subsurface storage provides several advantages over other possible alternatives of carbon sequestration. For subsurface carbon sequestration, there are at least four major mechanisms to trap the injected $\mathrm{CO}_{2}$ for long-term storage, namely, structural (stratigraphic) trapping, residual fluid trapping, solubility trapping, and mineral trapping. Some of these mechanisms may coexist and interplay simultaneously during the long span of storage process. A deep understanding of these trapping mechanisms and exploring possibly new trapping mechanisms require accurate mod-

*Submitted to the journal's Computational Methods in Science and Engineering section September 29, 2015; accepted for publication (in revised form) May 17, 2016; published electronically July 26, 2016. The research was supported in part by NSF China grants 11571100, 91530323 and 91530103. http://www.siam.org/journals/sisc/38-4/M104188.html

$\dagger$ College of Mathematics and Econometrics, Hunan University, Changsha, Hunan, 410082, China (haijianyang@gmail.com).

${ }^{\ddagger}$ Corresponding author. Institute of Software, Chinese Academy of Sciences, Beijing 100190, China, and State Key Laboratory of Computer Science, Chinese Academy of Sciences, Beijing 100190, China (yangchao@iscas.ac.cn).

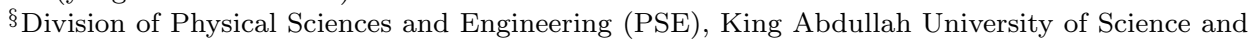
Technology (KAUST), Thuwal 23955-6900, Saudi Arabia (shuyu.sun@kaust.edu.sa). This author was supported by KAUST through the grant BAS/1/1351-01-01.

B593 
eling and simulation of two-phase and multiphase flow in subsurface formations. This paper is restricted to the discussion of two-phase flow, but the applied methodology is applicable to full compositional multiphase flow.

One basic requirement for accurate modeling and simulation of multiphase flow is to have the predicted saturation sit within a physically meaningful range, i.e., between zero and one. The saturation can be spatially and temporally varied within this range, but most algorithms used in practice do not guarantee the computed saturation will stay within this range. This is especially so when the applied computational method manifests numerical oscillation and nonphysical undershoot or overshoot [33]. A commonly used fix to this problem is to simply apply a cut-off operator to the computed saturation at each time step, i.e., to set the saturation to be zero whenever it becomes negative, and to set it to one whenever it becomes larger than one. This treatment, however, has a serious unpleasant consequence, which is the loss of mass conservation. Global and local mass conservation are desired properties for reservoir flow and other subsurface flow simulation. The majority of algorithms used in reservoir simulation have the property of local mass conservation, which of course implies also global conservation. Unfortunately, the applied cut-off operators not only destroy the local mass conservation but also damage the global mass conservation, which seriously ruins the numerical accuracy and physical interpretability of the simulation results.

It is safe to state that one major challenge in two-phase flow simulation is the nonlinearity of the process. Two main nonlinearities of two-phase flow are capillarity and relative permeability. Capillary pressure is one of main driving forces in fluid (oil, gas, and water) flow and transport in subsurface [37]. The capillarity effect can be the leading mechanism in oil recovery from fractured oil reservoirs. Capillarity is caused by surface tension between immiscible (or partially miscible) fluids. Relative permeability is a dimensionless measure of the effective permeability of a fluid phase, and it also plays an important role in enhanced oil recovery. In commonly used multiphase flow models, both the capillary pressure and relative permeability are nonlinear functions of saturation. They can be defined by interpolating experimental data or by using a closed-form formula, and they usually require the saturation to stay within a physically meaningful range, because otherwise the capillary pressure and relative permeability become undefined. As a result, it is important to ensure the saturation within a physically meaningful range, even from the viewpoint of treating capillarity and relative permeability.

A popular approach for solving two-phase flow problems is the implicit pressureexplicit saturation (IMPES) method [11, 12, 19, 59], in which the nonlinear problem is decoupled into two subproblems, namely, a linear equation for the potential component that needs to be solved implicitly and a nonlinear equation for the saturation variable that can be explicitly updated. However, the IMPES method often requires use of a small time step size to maintain a physical meaningful solution because of the stability restriction [13]. The situation becomes worse at the extreme scale due to the dependency between the time step size and the spatial resolution. Moreover, the semiimplicit nature of the algorithm may introduce a large splitting error due to the decoupling of the original problem [53]. Fully implicit methods [14, 36, 42, 51, 53, 54, 57], on the other hand, can relax the stability requirement on the time step size and therefore provide a consistent and robust way to solve the nonlinear problem in long-term and extreme-scale simulations. In particular, for problems with various time scales, fully implicit methods with a suitable time adaptivity strategy [44, 58] can lead to substantial reduction the total computing time, which greatly expands the scope of application of the fully implicit algorithm. 
When a fully implicit method is applied, one needs to solve a large-scale nonlinear algebraic system at each time step. Efforts have been made in employing the classical Newton method and its many variants to solve the two-phase flow problem [14, 36, 42]. However, numerical challenges arising from the boundedness of the solution still exist and may cause the Newton methods fail to converge. In this work, we introduce a variational inequality formulation of the saturation equilibrium with a box inequality constraint, which naturally satisfies the boundedness requirement. The two-phase variational inequality is then formulated as a nonlinear complementarity problem [21] which is solved by an active-set reduced-space method [3, 4, 22]. The active-set reduced-space method consists of two major steps: (a) in the first phase, an index set with respect to the computational domain is decomposed into active and inactive parts, based on a criterion specifying a certain active set method; and (b) in the second phase, a reduced linear system associated with the inactive set is solved. We would like to point out that the class of active-set reduced-space method has proven to be very efficient in a variety of applications $[23,28,34,35,56]$, but very little work has been done in the two-phase flow problem as far as we know.

For problems with high nonlinearities, preconditioning techniques on the nonlinear level, such as the additive Schwarz preconditioned inexact Newton (ASPIN) methods $[9,25,39]$, the nonlinear restricted Schwarz preconditioners [10, 16], the nonlinear dual-domain decomposition methods [48], the nonlinear balancing domain decomposition by constraints methods [30], the nonlinear elimination methods [26, 27, 29], and the composite nonlinear algebraic methods [6], have received increasing attention in recent years. In particular, some efforts have been made in applying the ASPIN method to solve the two-phase flow problems [51, 53]. Although the numerical results show that it is superior to the classical linear additive Schwarz method, the success of the ASPIN method depends strongly on an effective nonlinear subdomain solver, which limits the robustness of the algorithm, especially for the highly nonlinear models of two-phase flow problems. In this study, we present a nonlinear elimination algorithm as a nonlinear preconditioner to accelerate the convergence of the active-set reduced-space method. In the algorithm, the nonlinear elimination is carried out in a field-split manner to implicitly eliminate the solution components with high magnitude that often triggers the divergence of the nonlinear solver. We show by numerical experiments that the proposed algorithm is robust and efficient for a number of two-phase flow examples with strongly nonlinear relative permeabilities and spatially varied capillary pressure functions.

The paper is organized as follows. In section 2, we present a detailed description of the active-set reduced-space method with nonlinear elimination. In section 3 , a variational inequality based model of two-phase flow problems is introduced and then the proposed algorithm is applied to solve the resultant nonlinear systems. We carry out numerical experiments in section 4 to examine the efficiency and effectiveness of the proposed algorithm. The paper is concluded in section 5 .

2. Active-set reduced-space method with nonlinear elimination. The proposed active-set reduced-space method with nonlinear elimination consists of two major ingredients: (a) a global update based on an active-set reduced-space method as an outer nonlinear solve and (b) a subspace correction based on a field-split nonlinear elimination as a nonlinear preconditioner.

2.1. Active-set reduced-space methods. We first review the class of activeset reduced-space methods (see, e.g., $[2,45]$ ) for the numerical solution of large spare nonlinear variational inequality system of equations with lower and upper bounds, 
i.e., for a given nonlinear function $F: \mathbb{R}^{n} \rightarrow \mathbb{R}^{n}$, compute a vector $X \in \mathbb{R}^{n}$, such that

$$
\left\{\begin{array}{lll}
X_{i}=\phi_{i} & \text { and } & F_{i}(X)>0, \\
X_{i}=\psi_{i} & \text { and } & F_{i}(X)<0, \\
X_{i} \in\left[\phi_{i}, \psi_{i}\right] & \text { and } & F_{i}(X)=0,
\end{array}\right.
$$

where $F=\left(F_{1}, \ldots, F_{n}\right)^{T}, F_{i}=F_{i}\left(X_{1}, \ldots, X_{n}\right), X=\left(X_{1}, \ldots, X_{n}\right)^{T}$, and the inequality holds componentwisely by $\phi=\left(\phi_{1}, \ldots, \phi_{n}\right)^{T}$ and $\psi=\left(\psi_{1}, \ldots, \psi_{n}\right)^{T}$, i.e., only one of these three equations holds at a time. Let $\mathcal{S}=\{1,2, \ldots, n\}$ be an index set with each index corresponding to an unknown component $X_{i}$ and a nonlinear residual component $F_{i}$. Two special cases are the system of nonlinear equations, $F(X)=0$, obtained by taking $\phi_{i}=-\infty$ and $\psi_{i}=+\infty$ for each $i \in \mathcal{S}$, and the mixed nonlinear problem, where for some $i$ in the subset of $\mathcal{S}, \phi_{i}=-\infty$ and $\psi_{i}=+\infty$. In the study, we propose a class of active-set reduced-space methods for solving (2.1), which consists of the following steps: (a) in the first phase, the index set is decomposed into active and inactive parts, based on a criterion specifying a certain active set method; (b) in the second phase, a reduced linear system associated with the inactive set is solved, and then a line search method is applied to improve the efficiency of the iterative process.

The active and inactive sets used within the reduced-space method are defined as

$$
\left\{\begin{array}{l}
\mathcal{A}_{\phi}(X):=\left\{i \in \mathcal{S} \mid X_{i}=\phi_{i} \text { and } F_{i}(X)>0\right\} \\
\mathcal{A}_{\psi}(X):=\left\{i \in \mathcal{S} \mid X_{i}=\psi_{i} \text { and } F_{i}(X)<0\right\} \\
\mathcal{I}(X):=\mathcal{S} \backslash\left(\mathcal{A}_{\phi}(X) \cup \mathcal{A}_{\psi}(X)\right)
\end{array}\right.
$$

where $\mathcal{A}_{\phi}(X)$ and $\mathcal{A}_{\psi}(X)$ denote the active constraints at $X$ with respect to the lower bound $\phi$ and the upper bound $\psi$, respectively, and $\mathcal{I}(X)$ the inactive constraints. In fact, the active sets denote the variables where the lower and upper bounds are active and the function value can be ignored, i.e.,

$$
X_{i}=\left\{\begin{array}{l}
\phi_{i} \text { if } i \in \mathcal{A}_{\phi}(X), \\
\psi_{i} \text { if } i \in \mathcal{A}_{\psi}(X) .
\end{array}\right.
$$

The inactive set contains the remainder of the variables associated with a reduced linear system.

Starting from an initial guess $X^{0} \in \mathbb{R}^{n}$, suppose $X^{k}$ is the current approximate solution; then a new approximate solution $X^{k+1}$ can be computed through

$$
X^{k+1}=\pi\left[X^{k}+\lambda^{k} d^{k}\right]
$$

where $d^{k}$ is a search direction, $\lambda^{k}$ is the step length for line search, and the operator $\pi$ is a projection to cut off the undershoot or overshoot of the solution from the interval $[\phi, \psi]$, i.e., to set the component of the solution to be the lower bound whenever it becomes less than $\phi$, and to set it to $\psi$ whenever it becomes larger than the upper bound. To be more precise, at every iteration of the reduced-space method, the search direction $d^{k}$ includes three mutually disjoint subvectors $d_{\mathcal{A}_{\phi}^{k}}, d_{\mathcal{A}_{\psi}^{k}}$, and $d_{\mathcal{I}^{k}}$, with the first two corresponding to the active sets and the third to the inactive set. In the computation, we first set $d_{\mathcal{A}_{\phi}^{k}}$ and $d_{\mathcal{A}_{\psi}^{k}}$ to 0 , and then $d_{\mathcal{I}^{k}}$ is calculated by approximately solving the linear system equation

$$
\left(\nabla F\left(X^{k}\right)\right)_{\mathcal{I}^{k}, \mathcal{I}^{k}} d_{\mathcal{I}^{k}}=-F_{\mathcal{I}^{k}}\left(X^{k}\right),
$$




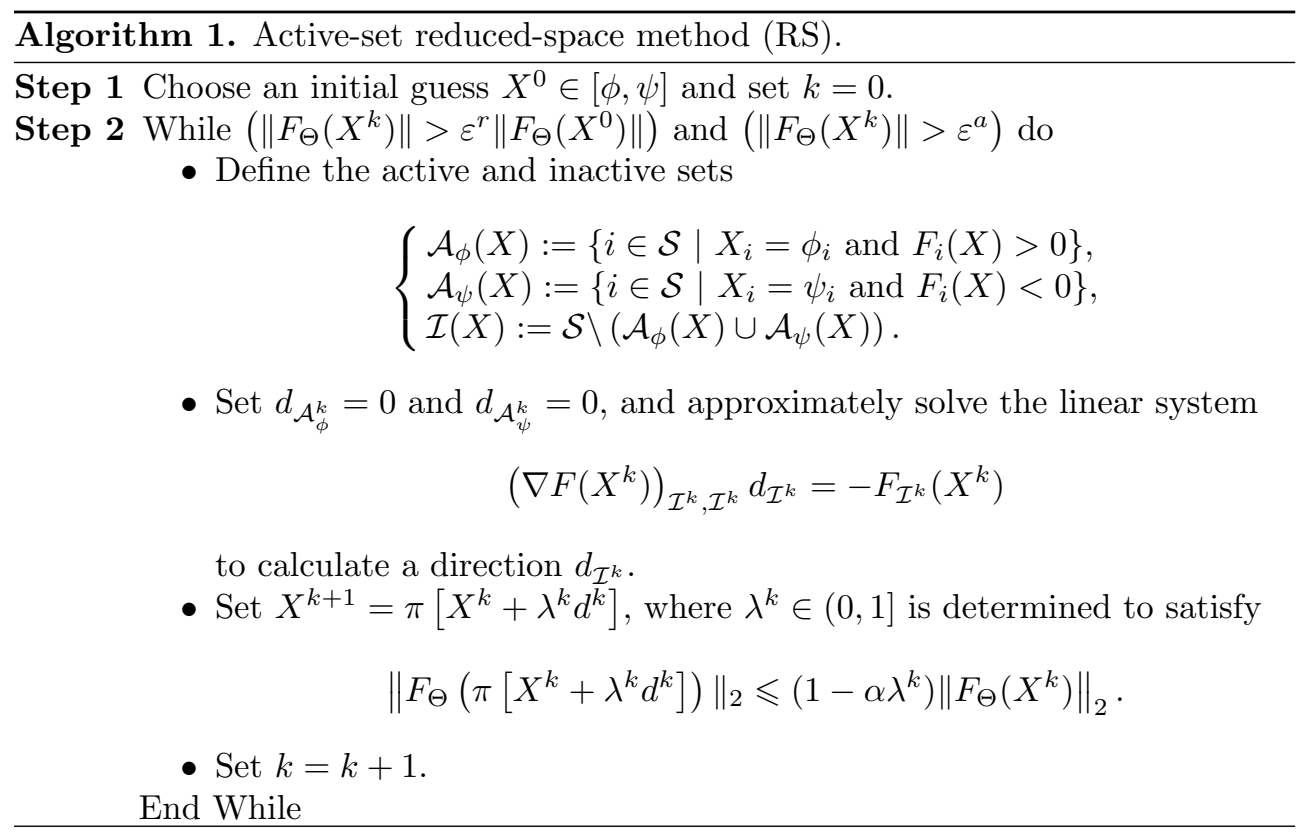

where $\left(\nabla F\left(X^{k}\right)\right)_{\mathcal{I}^{k}, \mathcal{I}^{k}}$ is a submatrix of $\nabla F\left(X^{k}\right)$ and $F_{\mathcal{I}^{k}}\left(X^{k}\right)$ is a subvector of $F\left(X^{k}\right)$, both based on the same index set $\mathcal{I}^{k}$. The step size $\lambda^{k}$ is chosen to be the optimal value for residual reduction with the constraint of

$$
\left\|F_{\Theta}\left(\pi\left[X^{k}+\lambda^{k} d^{k}\right]\right)\right\|_{2} \leqslant\left(1-\alpha \lambda^{k}\right)\left\|F_{\Theta}\left(X^{k}\right)\right\|_{2},
$$

where $\alpha$ is employed to ensure that the reduction of $\left\|F\left(X^{k}\right)\right\|_{2}$ is sufficient, and $F_{\Theta}(X)$ is a restriction operator of $F(X)$ defined componentwise as

$$
\left[F_{\Theta}(X)\right]_{i}= \begin{cases}F_{i}(X) & \text { if } \phi_{i}<X_{i}<\psi_{i}, \\ \min \left\{F_{i}(X), 0\right\} & \text { others. }\end{cases}
$$

Now the complete active-set reduced-space method (RS) algorithm is described in Algorithm 1.

In the RS method, we obtain a search direction $d^{k}$ by solving inexactly the reduced linear system (2.2), then compute the next approximate solution along this search direction. In (2.3), the damping scalar $\lambda^{k}$ is used to determine the step length that one should go in the selected search direction, and the parameter $\alpha$ is used to ensure that the reduction of the residual function $\left\|F_{\Theta}(X)\right\|_{2}$ is sufficient. In the ideal case, $\lambda^{k}=1$ is sufficient for RS, which means that a full step is taken. A near quadratic convergence may be observed, when the nonlinearities in the system are well-balanced. However, when the nonlinearity of the system is severely imbalanced, the size of $\lambda^{k}$ could be very small and the convergence becomes much slower. Observed from many numerical experiments, the slow convergence or sometimes divergence is often determined by the variables of equations in the system with the highest nonlinearities $[9,10,25]$. In our proposed algorithm, a nonlinear elimination step is applied as a subproblem solver inside the global RS iteration to smooth out the "high nonlinearity." Through subspace nonlinear solves, the high nonlinearities are removed, and the fast convergence can then be restored when the active-set reduced-space method is applied after the preconditioning. 
2.2. Nonlinear elimination. To define the nonlinear elimination (NE) preconditioner that is based on a field partition, the index set $\mathcal{S}$ is decomposed into two parts $\mathcal{S}_{b}$ and $\mathcal{S}_{g}$ corresponding to different physical or algorithmic components, i.e., $\mathcal{S}=\mathcal{S}_{b} \cup \mathcal{S}_{g}$. For notational convenience, we denote the vector $X=\left(X_{\mathcal{S}_{b}}, X_{\mathcal{S}_{g}}\right)$, and the nonlinear system $F(X)$ is rewritten as

$$
F(X)=F\left(X_{\mathcal{S}_{b}}, X_{\mathcal{S}_{g}}\right)=\left[\begin{array}{l}
F_{\mathcal{S}_{b}}\left(X_{\mathcal{S}_{b}}, X_{\mathcal{S}_{g}}\right) \\
F_{\mathcal{S}_{g}}\left(X_{\mathcal{S}_{b}}, X_{\mathcal{S}_{g}}\right)
\end{array}\right]=0
$$

For this partition, we define the subspace corresponding to the set $\mathcal{S}_{b}, V_{b} \subset \mathbb{R}^{n}$ as

$$
V_{b}=\left\{v \mid v=\left(v_{1}, \ldots, v_{n}\right)^{T} \in \mathbb{R}^{n}, v_{i}=0, \text { if } i \notin \mathcal{S}_{b}\right\},
$$

and the corresponding restriction operators $R_{b}$, which transfer a vector from $\mathbb{R}^{n}$ to $V_{b}$. Similarly, we define the subspace

$$
V_{g}=\left\{v \mid v=\left(v_{1}, \ldots, v_{n}\right)^{T} \in \mathbb{R}^{n}, v_{i}=0 \text {, if } i \notin \mathcal{S}_{g}\right\},
$$

and the corresponding operator $R_{g}$, which restrict the vector from $\mathbb{R}^{n}$ to $V_{g}$. Hence, using the restriction operator $R_{b}$, for any given $X \in \mathbb{R}^{n}$, we have

$$
F_{\mathcal{S}_{b}}(X)=R_{b}(F(X)) .
$$

Moreover, we define $\mathcal{T}_{b}(X): \mathbb{R}^{n} \rightarrow V_{b}$ as the solution of the following subspace nonlinear system:

$$
F_{\mathcal{S}_{b}}\left(R_{g}(X)+\mathcal{T}_{b}(X)\right)=0 .
$$

Using the subspaces mapping function, we introduce a new global function,

$$
Y=\mathcal{G}(X)=R_{g}(X)+\mathcal{T}_{b}(X) .
$$

2.3. General framework of RS-NE. The proposed nonlinear elimination preconditioned active-set reduced-space method can be seen as a class of nonlinear rightpreconditioning methods, i.e., the nonlinear elimination method is proposed for the construction of the inner nonlinear preconditioner and the classical active-set reducedspace method is used for the outer nonlinear solver. A high-level description of the basic algorithm for a general problem is presented in Algorithm 2.

The basic idea of RS-NE is to approximately eliminate the high nonlinearities before applying the global nonlinear iteration. Skipping the subspace correction phase, the proposed method is reduced to the classical RS method. Moreover, the RS method degrades into the classical inexact Newton method with backtracking [15, 17] for solving (2.1) when $\phi=-\infty$ and $\psi=+\infty$. Hence, for the mixed nonlinear problem, where for some $i$ in the subset of $\mathcal{S}, \phi_{i}=-\infty$ and $\psi_{i}=+\infty$, the proposed method can be considered as a class of coupled methods, i.e., the inexact Newton method with backtracking is used to solve the part of nonlinear system without restrictions, while the RS method is employed to solve the part of nonlinear system with restrictions. In Algorithm 2, a parameter $\epsilon_{\text {switch }}$, which is problem-dependent and requires the user to set, is employed as a switch to determine the timing of turning on or off the subspace correction phase. In addition, if the line search fails in the process of global nonlinear iterations, then the RS-NE method reverts to the subspace correction phase. 


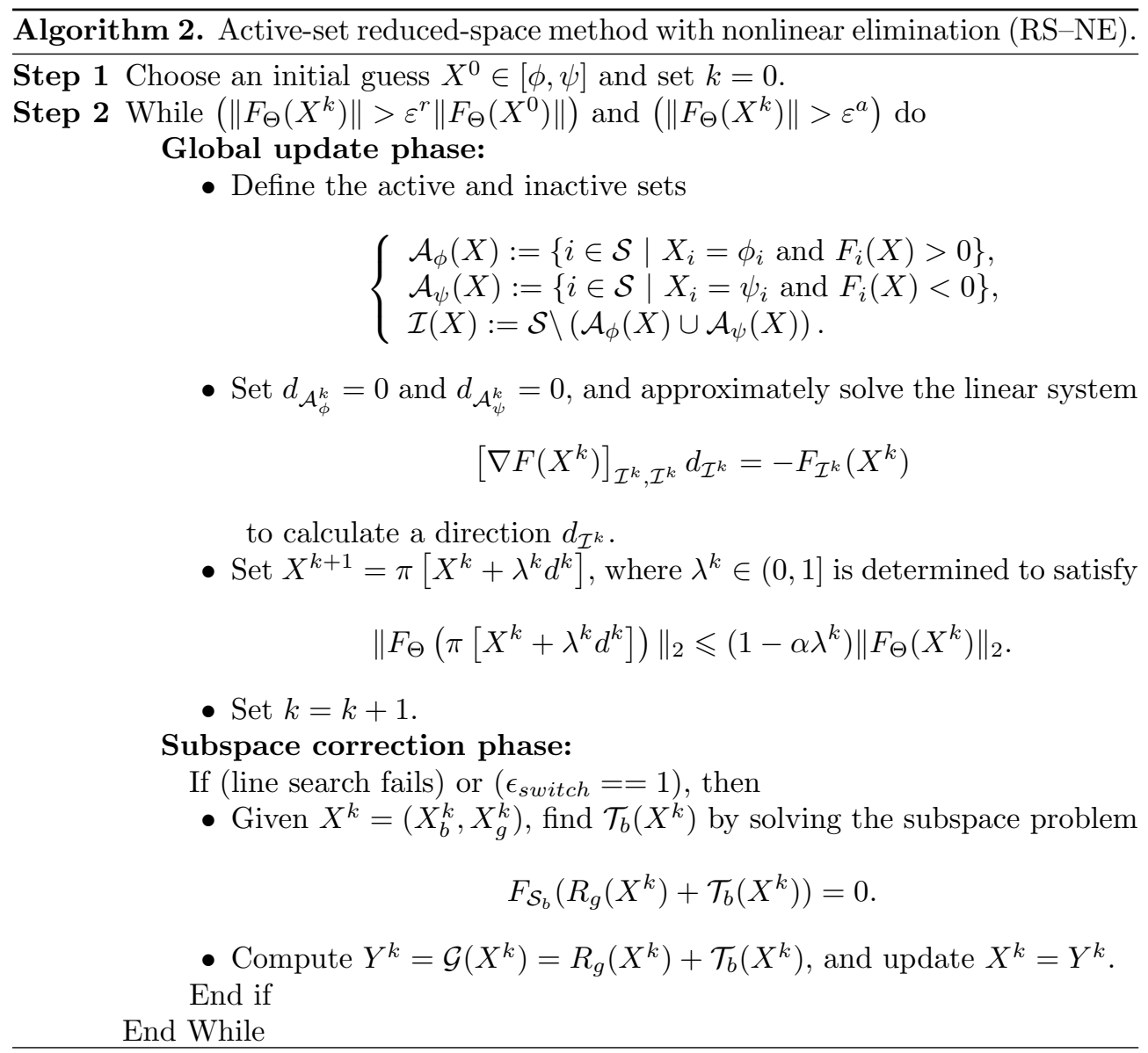

It is worth mentioning that the proposed nonlinear elimination method is based on the nonlinear function $F(X)$, rather than the variational inequality (2.1), to build the subspace systems (2.4) and (2.5). Moreover, the subspace problem (2.6), which is a reduced-space nonlinear system, can be solved using any classical nonlinear iterative method, such as the inexact Newton method with backtracking and its many variations $[7,31,46,47]$, and other composite solvers [6]. A key element of the NE step is the choice of $\mathcal{S}_{b}$ and $\mathcal{S}_{g}$ to build the subspaces $V_{g}$ and $V_{b}$. Some physics-based or field-based strategy can be used to determine the subset indices, and the partition of $\mathcal{S}_{b}$ and $\mathcal{S}_{g}$ for the nonlinear elimination may change with $k$. It differs from case to case. We also remark that the use of the nonlinear elimination preconditioner in this paper is different from the work in $[26,27]$ for the one- and two-dimensional scalar full potential equations. In [26, 27], the to-be-eliminated component is determined in advance based on certain knowledge of the problem, and thus the NE preconditioner is applied starting from the beginning until the intermediate solution is close to the desired solution. In our study, a class of nonlinear elimination methods as a right preconditioner is used for the general multicomponent systems, and the to-be-eliminated component is implicitly removed, based on the the feedback from the intermediate global nonlinear iterations. Hence, the NE preconditioner is activated only when the line search fails or the control parameter $\epsilon_{\text {switch }}$ is triggered in case that the global update is difficult to converge. 
3. RS-NE for two-phase flow in porous media. In this section, we introduce the continuous physical model together with the discretization employed for two-phase flow problems in porous media, and then present the implementation details of the RS-NE method for the resultant nonlinear systems.

3.1. Model problem. The flow consisting of two incompressible and immiscible fluids in porous media can be described by

$$
\left\{\begin{array}{l}
\phi \frac{\partial S_{\alpha}}{\partial t}+\nabla \cdot \mathbf{v}_{\alpha}=q_{\alpha}, \\
\mathbf{v}_{\alpha}=-\frac{k_{r \alpha} k}{\mu_{\alpha}}\left(\nabla p_{\alpha}+\rho_{\alpha} g \nabla z\right),
\end{array} \quad \alpha=w, n,\right.
$$

where $g$ is the gravity acceleration constant, $z$ is the depth, and $\phi$ and $k$ are the porosity and the absolute permeability of the media. Here the wetting phase of the flow is denoted by subscript $w$ and the nonwetting phase $n$. Each phase $\alpha$ has its own saturation $S_{\alpha}$, velocity $\mathbf{v}_{\alpha}$, pressure $p_{\alpha}$, density $\rho_{\alpha}$, viscosity $\mu_{\alpha}$, and relative permeability $k_{r \alpha}$. Later, we will show that the primary variables to be solved in (3.1) are $S_{w}$ and $p_{w}$. In this paper, the relative permeabilities are given by

$$
k_{r w}=S_{e}^{\beta}, \quad k_{r n}=\left(1-S_{e}\right)^{\beta},
$$

where $\beta$ is a positive integer number and $S_{e}$ is the normalized saturation. A commonly employed formulation of the normalized saturation is

$$
S_{e}=\frac{S_{w}-S_{r w}}{1-S_{r w}-S_{r n}},
$$

where $S_{r w}$ and $S_{r n}$ are the residual saturations for the wetting and nonwetting phases, respectively.

In (3.1), the first equation is determined by the mass conservation law, and the second one is the velocity equation following the Darcy's law. For the two-phase flow, the saturations of the phases are constrained by

$$
S_{w}+S_{n}=1
$$

and the pressures of the two phases are related through the capillary pressure function

$$
p_{c}\left(S_{w}\right)=p_{n}-p_{w}
$$

Throughout the study, we use the following nonlinear capillary pressure function [24]:

$$
p_{c}\left(S_{w}\right) \equiv-\frac{B_{c}}{\sqrt{k}} \log S_{e},
$$

where $B_{c}>0$ is related to the media property.

The heterogeneity of the capillary pressure in permeable media may result in discontinuity in the saturation, which in turn complicates the numerical simulation. For example, in the classical fractional flow formulation, contrast in capillary pressure may lead to discontinuous global pressure that makes the formulation inconsistent. In this paper, we follow the formulation in $[24,41]$. We first introduce the flow potential $\Phi_{\alpha}$ for phase $\alpha$ :

$$
\Phi_{\alpha}=p_{\alpha}+\rho_{\alpha} g z
$$


The capillary potential is then defined as

$$
\Phi_{c} \equiv \Phi_{n}-\Phi_{w}=p_{c}\left(S_{w}\right)+\left(\rho_{n}-\rho_{w}\right) g z .
$$

Further, we denote the mobility of phase $\alpha$ as $\lambda_{\alpha}=k_{r \alpha} / \mu_{\alpha}$. The total velocity can be rewritten into

$$
\mathbf{v} \equiv \mathbf{v}_{w}+\mathbf{v}_{n}=\mathbf{v}_{a}+\mathbf{v}_{c}
$$

with

$$
\mathbf{v}_{a}=-\lambda_{a} k \nabla \Phi_{w}, \quad \mathbf{v}_{c}=-\lambda_{n} k \nabla \Phi_{c} .
$$

Here the velocity $\mathbf{v}_{a}$ is associated with a mobility $\lambda_{a}=\lambda_{w}+\lambda_{n}$ that is smoother compared to either $\lambda_{w}$ or $\lambda_{n}$. The wetting-phase velocity can be recovered as $\mathbf{v}_{w}=f_{w} \mathbf{v}_{a}$ with $f_{w}=\lambda_{w} / \lambda_{a}$. Then, the potential equation of the two-phase flow is reformulated as

$$
\nabla \cdot\left(\mathbf{v}_{a}+\mathbf{v}_{c}\right)=q_{w}+q_{n}
$$

and the wetting-phase saturation equation becomes

$$
\phi \frac{\partial S_{w}}{\partial t}+\nabla \cdot\left(f_{w} \mathbf{v}_{a}\right)=q_{w},
$$

which holds on the set $0 \leqslant S_{w} \leqslant 1$.

Suppose the boundary of the computational domain $\Omega$ is composed of two parts $\partial \Omega=\Gamma^{D}+\Gamma^{N}$ with $\Gamma^{D} \cap \Gamma^{N}=\emptyset$. The boundary conditions associated to (3.4) and (3.5) are

$$
\begin{cases}p_{w}=p^{D} & \text { on } \Gamma^{D} \\ \mathbf{v} \cdot \mathbf{n}=q^{N} & \text { on } \Gamma^{N} \\ S_{w}=S^{N} & \text { on } \partial \Omega\end{cases}
$$

And the initial condition for the saturation equation is

$$
\left.S_{w}\right|_{t=0}=S_{w}^{0} \text { in } \Omega
$$

When using a fully implicit method, (3.4) and (3.5) are coupled and a nonlinear system is required to be solved at each time step. However, if we directly apply the Newton method or its many variations to solve such nonlinear systems, we face the numerical challenges arising from physically feasible saturation fractions between 0 and 1. Hence, we take the restriction of the saturation into account and introduce a variational inequality problem of the wetting-phase saturation equation (3.5). Considering a scaled $L^{2}$-inner product and the obstacle potential $0 \leqslant S_{w} \leqslant 1$, we obtain the following variational inequality for $(3.5)$; see $[4,5,22]$ for more details.

Problem 1. Given $S_{w}(\cdot, 0)=S_{w}^{0} \in H^{1}(\Omega)$ with $0 \leqslant S_{w}^{0} \leqslant 1$, find $S_{w} \in H^{1}\left(\Omega_{T}\right)$ such that $0 \leqslant S_{w} \leqslant 1$ a.e. in $\Omega_{T}=\Omega \times[0, T]$ and

$$
\left\langle\phi \frac{\partial S_{w}}{\partial t}, \mathcal{X}-S_{w}\right\rangle+\left\langle\nabla \cdot\left(f_{w} \mathbf{v}_{a}\right), \mathcal{X}-S_{w}\right\rangle-\left\langle q_{w}, \mathcal{X}-S_{w}\right\rangle \geqslant 0
$$

which has to hold for almost all $t$ and all $\mathcal{X} \in H^{1}(\Omega)$ with $0 \leqslant \mathcal{X} \leqslant 1$. Here $\langle\cdot, \cdot \cdot\rangle$ denotes the $L^{2}$-inner product. 
It is well known that the above problem is equivalent to the following problem:

$$
\begin{cases}S_{w}=0, \phi \frac{\partial S_{w}}{\partial t}+\nabla \cdot\left(f_{w} \mathbf{v}_{a}\right)-q_{w}>0 & \text { a.e. in } \Omega_{T}, \\ S_{w}=1, \phi \frac{\partial S_{w}}{\partial t}+\nabla \cdot\left(f_{w} \mathbf{v}_{a}\right)-q_{w}<0 & \text { a.e. in } \Omega_{T}, \\ S_{w} \in[0,1], \phi \frac{\partial S_{w}}{\partial t}+\nabla \cdot\left(f_{w} \mathbf{v}_{a}\right)-q_{w}=0 & \text { a.e. in } \Omega_{T},\end{cases}
$$

where all equalities and inequalities have to hold almost everywhere. Equation (3.8) is known as a box constrained variational inequality or mixed complementarity problem. For more details, we recommend [18, 20, 21, 45, 49] and references therein.

For the purpose of comparison, an IMPES method is also implemented. The semi-implicit scheme comprises two split steps: one implicitly solves the potential equation (3.4) to obtain a new potential of the wetting phase $\Phi_{w}$ and thus $\mathbf{v}_{a}$ from (3.3), and the other explicitly updates the wetting-phase saturation (3.5).

3.2. Discretization. In this study, a cell-centered finite difference (CCFD) is applied to spatially discretize the optimization problem for the spatial terms; the detailed process of CCFD can be found [32, 40,42]. The employed CCFD discretization for the pressure equation in rectangular meshes can also be viewed as the mixed finite element method with Raviart-Thomas basis functions of lowest order equipped with the trapezoidal quadrature rule. We remark here that the proposed nonlinearly preconditioned solutions algorithms can be generalized to the case of other spatial discretizations.

Let $\Omega$ be the computational domain covered with $N_{x} \times N_{y}$ mesh cells. Then each mesh point $p_{i, j}=\left(x_{i}, y_{j}\right)$ is centered at the position $x_{i}=i \times h_{x}$ and $y_{j}=j \times h_{y}$ with $i=1, \ldots, N_{x}, j=1, \ldots, N_{y}, h_{x}=1 / N_{x}$, and $h_{y}=1 / N_{y}$. For a given time-stepping sequence $0=t^{(0)}<t^{(1)}<t^{(2)}<\cdots$, define the time step size $\Delta t^{(l)}=t^{(l+1)}-t^{(l)}$ and use superscript $(l)$ to denote the discretized evaluation at time level $t=t^{(l)}$. Then, the discretization of the saturation equation in (3.5) is

$$
F_{S_{w}}\left(S_{w}^{(l+1)}, p_{w}^{(l+1)}\right)=\phi \frac{S_{w}^{(l+1)}-S_{w}^{(l)}}{\Delta t^{(l)}}+\nabla_{h}^{*} \cdot\left(f_{w}^{(l+1)} \mathbf{v}_{a}^{(l+1)}\right)-q_{w}^{(l+1)}=0,
$$

where $\nabla_{h}^{*}$. represent CCFD discretization of the gradient operator by using the upwind scheme according to the velocity $\mathbf{v}_{a}^{(l+1)}$. Hence, the discretization of variational inequality (3.8) is given as

$$
\begin{cases}S_{w}^{(l+1)}=0 & \text { and } \quad F_{S_{w}}\left(S_{w}^{(l+1)}, p_{w}^{(l+1)}\right)>0 \\ S_{w}^{(l+1)}=1 & \text { and } \quad F_{S_{w}}\left(S_{w}^{(l+1)}, p_{w}^{(l+1)}\right)<0 \\ S_{w}^{(l+1)} \in[0,1] \quad \text { and } & F_{S_{w}}\left(S_{w}^{(l+1)}, p_{w}^{(l+1)}\right)=0\end{cases}
$$

Similarly, we employ a CCFD scheme for (3.4); then the discretization of the potential equation is 


$$
\begin{aligned}
F_{p_{w}}\left(S_{w}^{(l+1)}, p_{w}^{(l+1)}\right) & =-\nabla_{h} \cdot \lambda_{a}^{(l+1)} \nabla_{h} \Phi_{w}^{(l+1)}-\nabla_{h} \cdot \lambda_{n}^{(l+1)} \nabla_{h} \Phi_{c}^{(l+1)}-q_{w}^{(l+1)}-q_{n}^{(l+1)} \\
& =0
\end{aligned}
$$

where $\nabla_{h}$. and $\nabla_{h}$ represent CCFD discretizations of the divergence and gradient operators. We remark here that the CCFD scheme conserves the mass both globally and locally because of the consistent discretization of the local differential operator across all mesh cell interfaces.

3.3. The application of RS-NE to two-phase flow. In this subsection, we introduce the implementation details of the RS-NE method for two-phase flow. In the implementation, we use the fully coupled ordering to build up the nonlinear system (2.1), by which we mean that different physical variables defined at the same grid point are always put together throughout the calculations. At each grid point, we arrange the unknowns in the order of $\left(p_{w}\right)_{i j}$ and $\left(S_{w}\right)_{i j}$, and then all grid points numbered in the natural ordering. That is, the unknowns are ordered in the order of

$$
X=\left(\left(p_{w}\right)_{11},\left(S_{w}\right)_{11}, \ldots,\left(p_{w}\right)_{i j},\left(S_{w}\right)_{i j}, \ldots,\left(p_{w}\right)_{N_{x} N_{y}},\left(S_{w}\right)_{N_{x} N_{y}}\right) \in \mathbb{R}^{n},
$$

where $n=2 N_{x} N_{y}$. The corresponding functions are ordered in the order of

$$
F(X)=\left(\left(F_{p_{w}}\right)_{11},\left(F_{S_{w}}\right)_{11}, \ldots,\left(F_{p_{w}}\right)_{i j},\left(F_{S_{w}}\right)_{i j}, \ldots,\left(F_{p_{w}}\right)_{N_{x} N_{y}},\left(F_{S_{w}}\right)_{N_{x} N_{y}}\right) \in \mathbb{R}^{n} .
$$

The lower bound in (2.1) is given as

$$
\phi=(-\infty, 0, \ldots,-\infty, 0, \ldots,-\infty, 0) \in \mathbb{R}^{n},
$$

and the upper bound is

$$
\psi=(+\infty, 1, \ldots,+\infty, 1, \ldots,+\infty, 1) \in \mathbb{R}^{n} .
$$

In the NE step, we use a field-based strategy to determine $\mathcal{S}_{b}$ and $\mathcal{S}_{g}$ and propose the inexact Newton method with backtracking for solving the subspace problem (2.6). In the following, we take the wetting-phase saturation approach (i.e., removing the $S_{w}$-component) as an example to build the NE step. In the approach, we consider all the wetting-phase saturation $S_{w}$ as bad components. Following the order in (3.12), the index set $\mathcal{S}_{b}$ with respect to the component $S_{w}$ is all the even numbers of the set $\mathcal{S}$, and $\mathcal{S}_{g}=\mathcal{S} \backslash \mathcal{S}_{b}$. Also, we can define the corresponding subspaces $V_{b}$ and $V_{g}$ in (2.4) and (2.5), respectively. Then,

$$
Y_{i}= \begin{cases}X_{i} & \text { if } i \in \mathcal{S}_{g} \\ \left(\mathcal{T}_{b}(X)\right)_{i} & \text { if } i \in \mathcal{S}_{b}\end{cases}
$$

where $\mathcal{T}_{b}$ is the solution of subspace nonlinear system (2.6) with the inexact Newton method with backtracking. To active the NE step, we need to set the parameter $\epsilon_{\text {switch }}=1$ when necessary. The strategy we use here is as follows. We first take several (controlled by a threshold $N_{\text {switch }}$, unless the line search fails) global nonlinear iterations using the RS method, then solve the subspace problem by the inexact Newton method with backtracking, and this process continues until convergence. 
A major advantage of fully implicit schemes is that the time step size $\Delta t$ is no longer constrained by the CFL condition, which is often required by explicit or semi-implicit methods. Meanwhile, for the simulation of two-phase flow, the evolution of the system usually admits various time scales and the calculation often lasts for a long time. Hence, we can use an adaptive time step control to enhance the temporal accuracy and reduce the total computing time. In the study, analogous to the switched evolution/relaxation strategy in [44], we start with a relatively small time step size $\triangle t^{(0)}$ and update its value via $\triangle t^{(l+1)}=\beta^{(l)} \Delta t^{(l)}$ with $\beta^{(l)}$ being the scaling factor of the adjacent time step size

$$
\beta^{(l)}=\max \left(\frac{1}{\rho}, \min \left(\rho,\left(\frac{\left\|F\left(X^{(l)}\right)\right\|_{2}}{\left\|F\left(X^{(l+1)}\right)\right\|_{2}}\right)^{\eta}\right)\right),
$$

where $\left\|F\left(X^{(l+1)}\right)\right\|_{2}$ is the Euclidean norm of $F\left(X^{(l+1)}\right)$. In the formulation, $\rho \in$ $(0,+\infty)$ is a safeguard to avoid excessive change of the time step size between any two immediate time steps, and $\eta \in(0,1]$ is used to control the adjustment of the time step size.

4. Numerical experiments. In this section, we report some results of numerical experiments to examine the performance of both traditional and the proposed algorithms listed as follows:

- IMPES denotes the implicit pressure-explicit saturation method.

- INB denotes the inexact Newton method with backtracking.

- RS denotes the classical active-set reduced-space method.

- RS-NE denotes the active-set reduced-space method with nonlinear elimination.

- Adaptive RS-NE denotes the nonlinear elimination preconditioned active-set reduced-space method with adaptive time stepping.

All these algorithms are implemented based on the Portable, Extensible Toolkits for Scientific computation (PETSc) [1] library. We test the algorithms on a Dell PowerEdge C6100 supercomputer that contains two hex-core 2.8-GHz Intel Westmere processors and 24 GB local memory in each node and equipped with a nonblocking QDR Infiniband high performance network. It is worth mentioning that all algorithms except the IMPES method are employed in conjunction with the fully implicit method. There are several nonlinear and linear iterative procedures in the proposed algorithms, and each requires a proper stopping condition. In the RS method, an absolute (relative) tolerance of $10^{-3}\left(10^{-8}\right)$ is utilized for the global nonlinear iteration, and the absolute (relative) tolerance is set to $10^{-10}\left(10^{-6}\right)$ for the subspace nonlinear iteration. In nonlinear iterations, we use the standard cubic backtracking algorithm $[15,17]$ with $\alpha=10^{-4}$ to pick the step length $\lambda$. In the IMPES method, the time step subdivision for saturation is 5 . All linear systems are solved by the Schwarz preconditioned GMRES method $[8,50,52,55]$, with relative and absolute tolerances of $10^{-3}$ and $10^{-6}$, respectively. The subdomain problems in the Schwarz preconditioner are solved with the sparse LU factorization. Moreover, in the adaptive RS-NE method we use $\rho=2.5$ and $\eta=0.75$ to control the time step size $\triangle t$, if not explicitly stated.

In the study, we use five test cases for two-phase fluid flows in a horizontal layer consisting of regular heterogeneous permeability [24, 32, 53]. In all cases, with the porous media being horizontal, the effect of gravity is neglected, and the void of the medium is initially fully saturated with oil, i.e., the initial conditions are $p_{w}=0$ and 
TABLE 1

Parameters for all test cases.

\begin{tabular}{|l|l|l|l|l|l|}
\hline Parameters & Case-1 & Case-2 & Case-3 & Case-4 & Case-5 \\
\hline Computational domain (m) & $300 \times 150$ & $300 \times 150$ & $300 \times 150$ & $500 \times 270$ & $100 \times 100$ \\
$\phi$ & 0.2 & 0.2 & 0.2 & 0.2 & 0.2 \\
$k$ (md) & 1 or 100 & 1,50 or 100 & 1 or 100 & 1 or 100 & $0.03-400$ \\
$\mu_{w}(\mathrm{cp})$ & 1 & 1 & 1 & 1 & 1 \\
$\mu_{n}(\mathrm{cp})$ & 0.45 & 0.25 & 0.5 & 0.45 & 0.2 \\
$\beta$ & 2 & 2 & 2 & 2 & 2 \\
$B_{c}\left(\right.$ bar md $\left.^{1 / 2}\right)$ & 70 & 30 & 100 & 50 & 25 \\
$S_{r w}$ & 0 & 0 & 0 & 0 & 0 \\
$S_{r n}$ & 0 & 0 & 0.2 & 0 & 0 \\
Injection rate (PV/year) & 0.15 & 0.11 & 0.11 & 0.1 \\
\hline
\end{tabular}

$S_{w}=S_{m}$, where $S_{m}=10^{-4}$ is the minimum of saturation. We assume the top and bottom boundaries of the reservoir to be impermeable, i.e., the normal component of the Darcy velocity on these boundaries vanishes. Then the system is flooded by water from left to right, i.e., we set $\frac{\partial p_{w}}{\partial \mathbf{n}}=0, S_{w}=1-S_{m}$ at the left boundary and set $p_{w}=0, \frac{\partial S_{w}}{\partial \mathbf{n}}=0$ at the right boundary. There is no injection/extraction to the interior of the domain. Table 1 summarizes the main features of each test case, including the domain size, permeability, and porosity data. The purposes of the tests include (a) the verification of the numerical accuracy of the fully implicit method; (b) a comparison of the performance of the IMPES method and the RS-NE method; (c) a comparison of different fully implicit solvers, including the classical INB and RS methods and the RS-NE method with adaptive time stepping; (d) a comparison of different parameters for RS-NE with adaptive time stepping; (e) the parallel performance of the fully implicit method.

4.1. Numerical validation. We first validate the discretization scheme and the fully implicit solver by running Case-1. As shown in Figure 1, the computational domain for Case- 1 is 300 meters long by 150 meters wide and includes two kinds of permeabilities (1 md and $100 \mathrm{md}$ ) in subdomains, respectively, i.e.,

$$
k= \begin{cases}100 \mathrm{md} & 70 \leqslant x \leqslant 150 \text { and } 50 \leqslant y \leqslant 100 \\ 1 \mathrm{md} & \text { otherwise }\end{cases}
$$

The saturation is calculated until 0.5 pore volume injection (PVI), and the wetting phase is injected with the flow rate $0.15 \mathrm{PV} /$ year from the left boundary, i.e., the simulation time is ended at $t=0.5 / 0.15$ year. In this test, we use a fixed time step size $\triangle t=0.1$ year (i.e., 36.5 days) for the RS-NE method and use $\triangle t=5 \times 10^{-4}$ year (i.e., 0.1825 day) for the IMPES method. Numerical results of both the IMPES method and the fully implicit method on a $100 \times 50$ mesh are shown in Figure 1, which verifies the consistency between the semi-implicit and the fully implicit methods. We remark that the IPMES method cannot obtain the smooth results when the time step size $\triangle t$ is larger than $6 \times 10^{-4}$ year, which is consistent with [32].

We further run Case-2 to verify the robustness of the fully implicit method. Compared to the previous test case with two different permeabilities, in Case-2 there are three subdomains with the permeabilities of $1 \mathrm{md}, 50 \mathrm{md}$, and $100 \mathrm{md}$, as shown in the top panel of Figure 2. We run the test on a $100 \times 50$ mesh using the fully implicit and semi-implicit methods. In the simulation, we again use a fixed time step size $\triangle t=0.1$ year for the fully implicit method and use $\triangle t=5 \times 10^{-4}$ year for the IMPES method. The wetting phase is injected with the flow rate $0.11 \mathrm{PV} /$ year from 

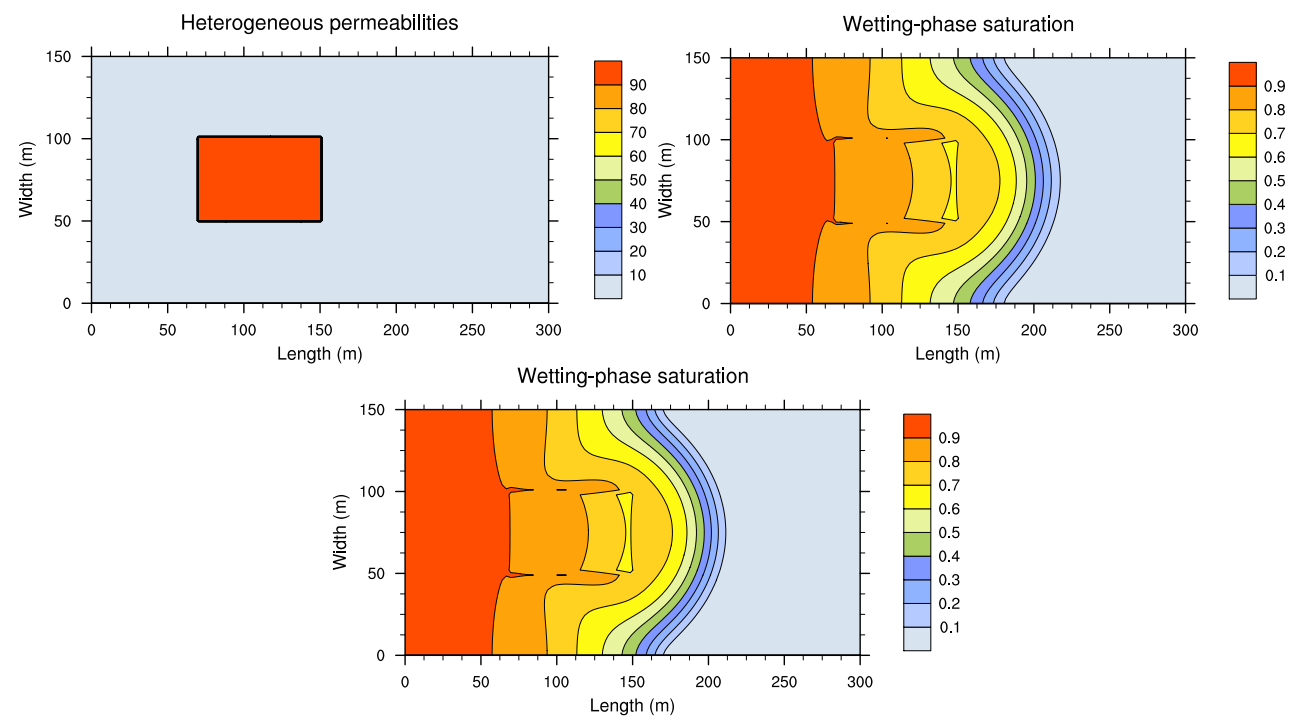

FIG. 1. Case-1: Heterogeneous permeabilities and wetting-phase saturation profiles at 0.5 PVI. The mesh is $100 \times 50$. The top-left panel shows the distribution of the heterogeneous permeabilities; the top-right panel shows the contour plot of the wetting-phase saturation by using $R S-N E$ with $\triangle t=0.1$ year; the bottom panel shows the contour plot of the wetting-phase saturation by using IMPES with $\triangle t=5 \times 10^{-4}$ year.
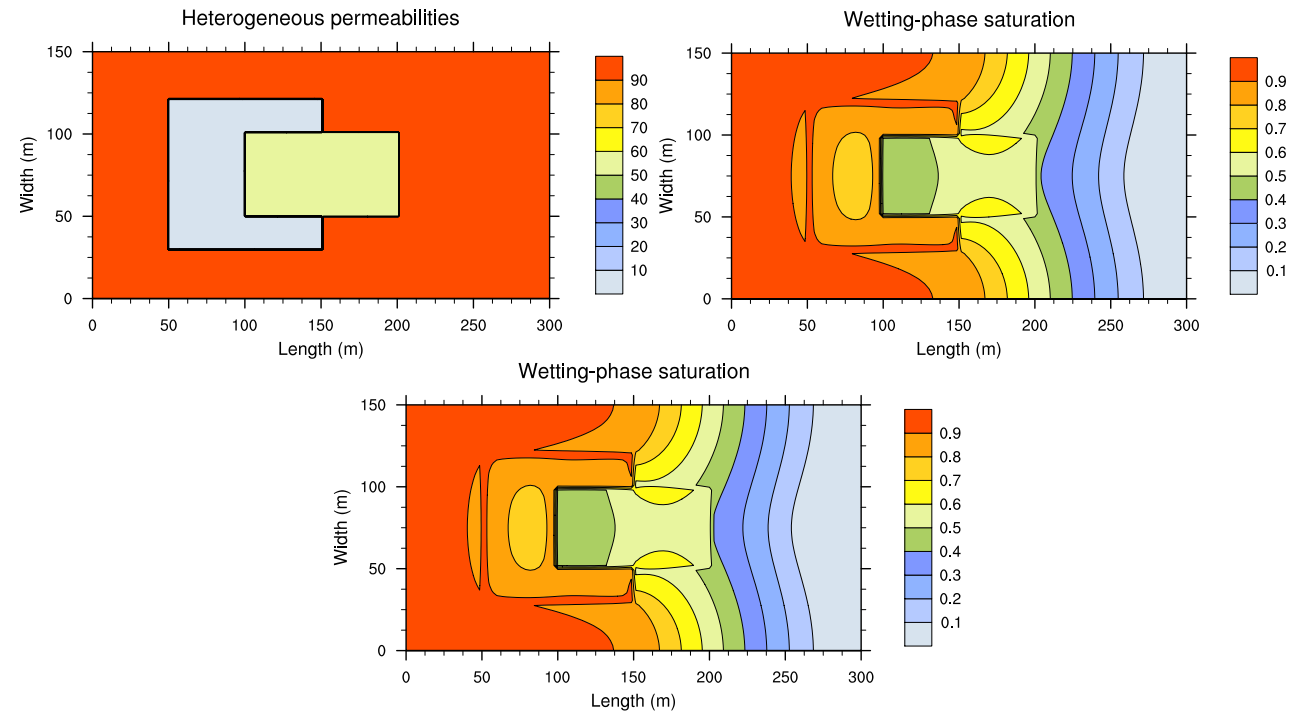

FIG. 2. Case-2: Heterogeneous permeabilities and wetting-phase saturation profiles at 0.6 PVI. The mesh is $100 \times 50$. The top-left panel shows the distribution of the heterogeneous permeabilities; the top-right panel shows the contour plot of the wetting-phase saturation by using RS-NE with $\triangle t=0.1$ year; the bottom panel shows the contour plot of the wetting-phase saturation by using IMPES with $\triangle t=5 \times 10^{-4}$ year.

the left boundary. The wetting-phase saturation profiles as 0.5 PVI computed by the two methods are presented in the bottom panel of Figure 2. The results illustrate that the new method can yet compute a reasonable solution with a much larger time step size, when compared with the IMPES approach. 

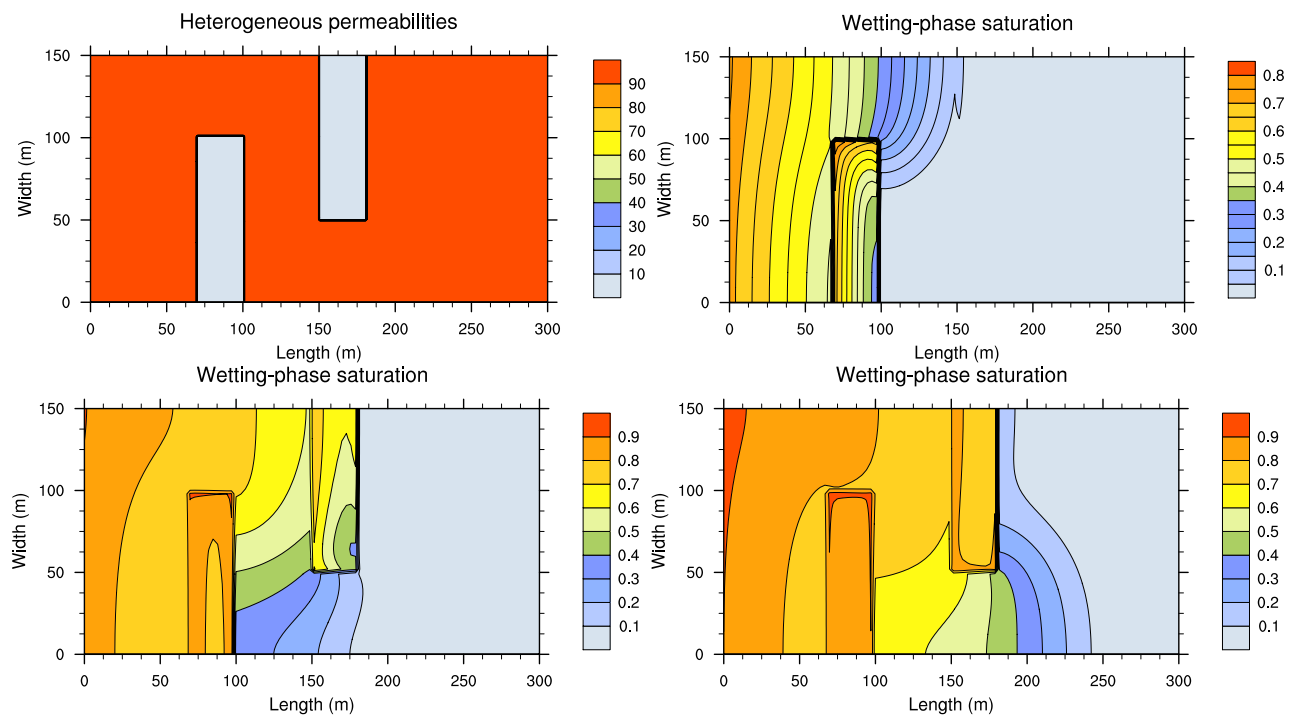

FIG. 3. Case-3: Heterogeneous permeabilities and wetting-phase saturation profiles by using RSNE. The mesh is $100 \times 50$. The top-left panel is for the heterogeneous permeabilities; the top-right panel is for wetting-phase saturation profiles at $0.2 \mathrm{PVI}$; the bottom-left panel is for wetting-phase saturation profiles at 0.4 PVI; the bottom-right panel is for wetting-phase saturation profiles at 0.5 $P V I$.

Next, we compare the RS-NE method with the IMPES method for Case-3 and Case- 4 in terms of time step size and total compute time. In RS-NE, we consider its two different versions, i.e., the standard and adaptive RS-NE methods. In Case-3, the tested medium consists of three subdomains with the different permeabilities of 1 md or $100 \mathrm{md}$, i.e.,

$$
k= \begin{cases}1 \mathrm{md}, & 70 \leqslant x \leqslant 100 \text { and } 0 \leqslant y \leqslant 100 \\ 1 \mathrm{md}, & 150 \leqslant x \leqslant 180 \text { and } 50 \leqslant y \leqslant 150 \\ 100 \mathrm{md} & \text { otherwise }\end{cases}
$$

The configurations are shown in the top-left panel of Figure 3. The porosity of this medium $\phi$ is 0.2 . The relative permeabilities are quadratic function of water saturation, i.e., $\beta=2$. The capillary pressure parameter $B_{c}$ is taken as 100 bar. The injection rate is $0.2 \mathrm{PV} /$ year. Other relevant parameters are provided in Table 1. We run the test on a $100 \times 50$ mesh using RS with a fixed time step size $\Delta t=$ 0.02 year. In Figure 3, we show the contour plots of the calculated wetting-phase saturation at $t=0.2,0.4$, and 0.5 PVI. We can see that the fully implicit approach successfully resolves the evolution of the wetting-phase saturations, and we find that the resemblance between the simulated results and the published results in [32] is remarkable.

In this case, the domain is highly heterogeneous and the capillary pressure functions differ on the different subdomains, leading to the increase in nonlinearity of the problem that affects the size of the time step. In Table 2, we compare the fully implicit method with the semi-implicit method in terms of time step sizes and the computing time. The time step size of IMPES is selected as $\triangle t=2.5 \times 10^{-4}$ such that it satisfies the requirement of stability. In the adaptive RS-NE method, we set the initial time step size to be $\Delta t=0.01$ and then adaptively control it by using (3.14). 
TABLE 2

A comparison of RS-NE and IMPES for Case-3. The mesh is $100 \times 50$ on four processors. The simulation is finished at 0.5 PVI. In the adaptive $R S-N E$ method, we set the initial time step size to be $\triangle t=0.01$.

\begin{tabular}{|l|c|c|c|}
\hline & IMPES & RS-NE & Adaptive RS-NE \\
\hline Number of time steps & $10^{4}$ & 125 & 42 \\
Average time step size (years) & $2.5 \times 10^{-4}$ & 0.02 & 0.061 \\
Average nonlinear iteration & -- & 6.4 & 12.4 \\
Execution time (second) & 137.4 & 54.7 & 30.9 \\
\hline
\end{tabular}

In the standard RS-NE method, the time step size is fixed to $\triangle t=0.02$. We would like to emphasize that the IPMES method and the standard RS-NE method cannot obtain smoothed solutions when the time step size becomes larger. It is clear from Table 2 that the required number of time steps of RS-NE is much smaller than that of IMPES. Moreover, we can take a larger time step size for the RS-NE method when an adaptivity mechanism is used to control the time step size, and in this case, the execution time can be further reduced, although the averaged number of nonlinear iterations increases. This indicates the new method has better stability and efficiency than the IMPES method.

In the following, we compare the RS-NE method with the IMPES method for Case-4. In Case-4, as shown in Figure 4, the domain is also composed of layers of alternate permeabilities ( $1 \mathrm{md}$ and $100 \mathrm{md})$, i.e.,

$$
k= \begin{cases}1 \mathrm{md}, & 30+60 i \leq y \leq 60+60 i, i=0,1,2,3, \\ 100 \mathrm{md} & \text { otherwise }\end{cases}
$$

The wetting phase is uniformly injected across the left-hand side of the layered domain, which is initially saturated with the nonwetting phase. The production is across the opposite right-hand side. The injection rate is $0.11 \mathrm{PV} /$ year. Other relevant parameters are again given in Table 1. In Figure 4, we show the contour plots of the calculated wetting-phase saturation for the test case at different PVI. As shown in the figures, the flow behavior with homogeneous capillary pressure is essentially influenced by the low permeability field and causes a great delay of displacement of the layer with the smaller permeability value.

We then compare the performance in terms of time step sizes and the computing time in Table 3. In the adaptive RS-NE method, we again set the initial time step size to be $\triangle t=0.01$ year and then adaptively control it by using (3.14). In the IMPES method, the time step size is changed to $\triangle t=5 \times 10^{-4}$ year. In the standard RS-NE method, the time step size is changed to $\Delta t=0.03$ year. From Table 3 , it is clear that the time step size of the fully implicit method is far larger than that of IMPES, and the total computing time of the fully implicit method is also much smaller than that of the semi-implicit method. We remark that even with large time step sizes, the fully implicit method with adaptive time stepping has a similar accuracy as the semi-implicit method and the fully implicit method with a fixed, small time step size.

From the above tests, we conclude that the RS-NE method, especially the adaptive time-stepping approach, is faster and more robust than the IMPES method in terms of time step sizes and the computing time. We would like to emphasize that, with refined spatial resolution, the advantage of the fully implicit method with adaptive time stepping becomes increasingly pronounced, as seen in the weak scaling tests to be discussed in the last subsection. 

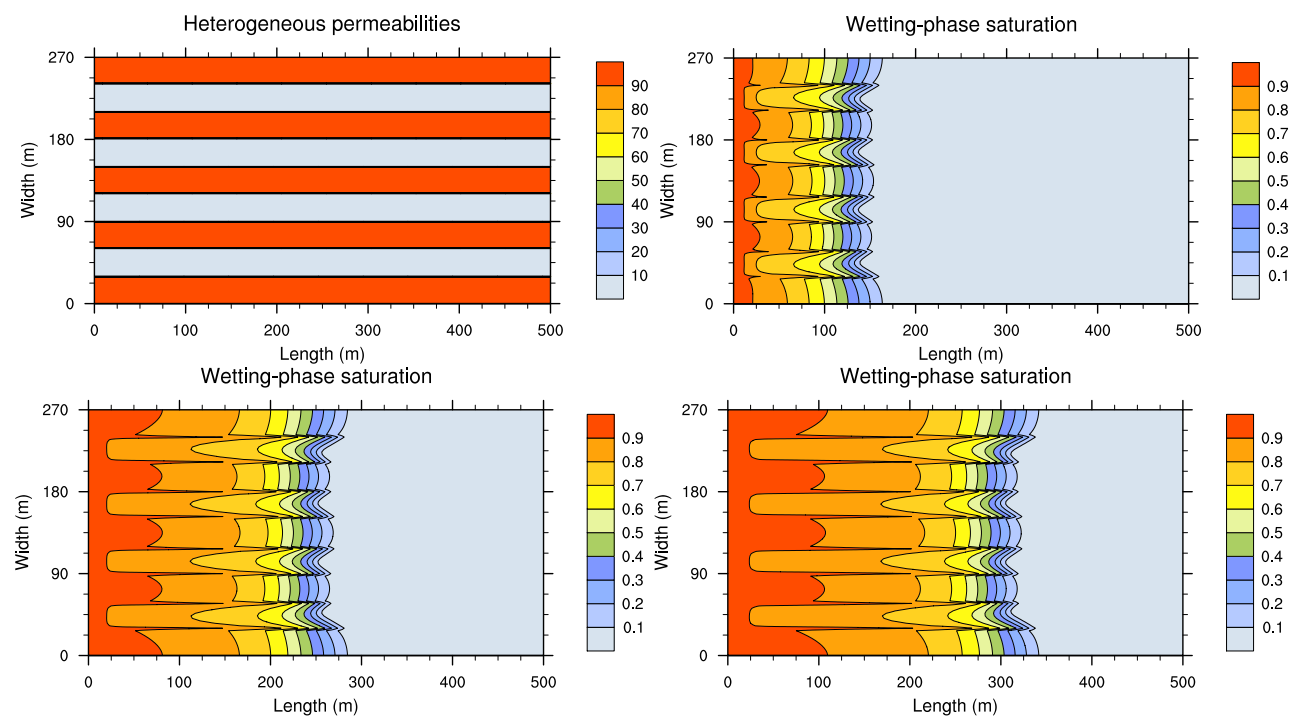

FIG. 4. Case-4: Heterogeneous permeabilities and wetting-phase saturation profiles at different times. The mesh is $200 \times 100$. The top-left panel is for the heterogeneous permeabilities; the top-right panel is for wetting-phase saturation profiles at 0.2 PVI; the bottom-left panel is for wetting-phase saturation profiles at 0.4 PVI; the bottom-right panel is for wetting-phase saturation profiles at 0.6 $P V I$.

TABLE 3

A comparison of RS-NE and IMPES for Case-4. The mesh is $200 \times 100$ on four processors. The simulation is $0.6 \mathrm{PVI}$. In the adaptive $R S-N E$ method, we set the initial time step size to be $\triangle t=0.01$.

\begin{tabular}{|l|c|c|c|}
\hline & IMPES & RS-NE & Adaptive RS-NE \\
\hline Number of time steps & 10909 & 182 & 75 \\
Average time step size (years) & $5 \times 10^{-4}$ & 0.03 & 0.0735 \\
Average nonlinear iteration & -- & 5.7 & 10.4 \\
Execution time (second) & 925.1 & 630.3 & 397.9 \\
\hline
\end{tabular}

Finally, we test the adaptive RS-NE method with a more difficult test case, in which the permeabilities of the porous medium are random. The configuration of the test case is listed as Case- 5 in Table 1. In the test, the permeabilities of the porous medium are generated by a geostatistical model using the open source code MRST (MATLAB Reservoir Simulation Toolbox) [38], ranging from 0.03 to $400 \mathrm{md}$. The heterogeneity of the permeabilities often causes unexpected early water breakthrough and is challenging for the numerical techniques. In the test we set the initial time step to $\triangle t=0.02$ and run the simulation on a $100 \times 100$ mesh. The contour plots of wetting-phase saturation profiles by using the adaptive RS-NE method at different times are shown in Figure 5. We can see that the proposed approach successfully resolves the rapid and abrupt evolution of the wetting-phase saturation while keeping it within the physically meaningful range.

4.2. Comparison of fully implicit methods. In this subsection, we restrict ourselves to the class of fully implicit methods for Case- 3 and Case- 4 . In the tests, we first study some parameters that impact the performance of the adaptive RS-NE method. These parameters include the threshold $N_{\text {switch }}$ defined in subsection 3.3 for the nonlinear iterations and the adaptivity parameters $\rho$ and $\eta$ for controlling the 

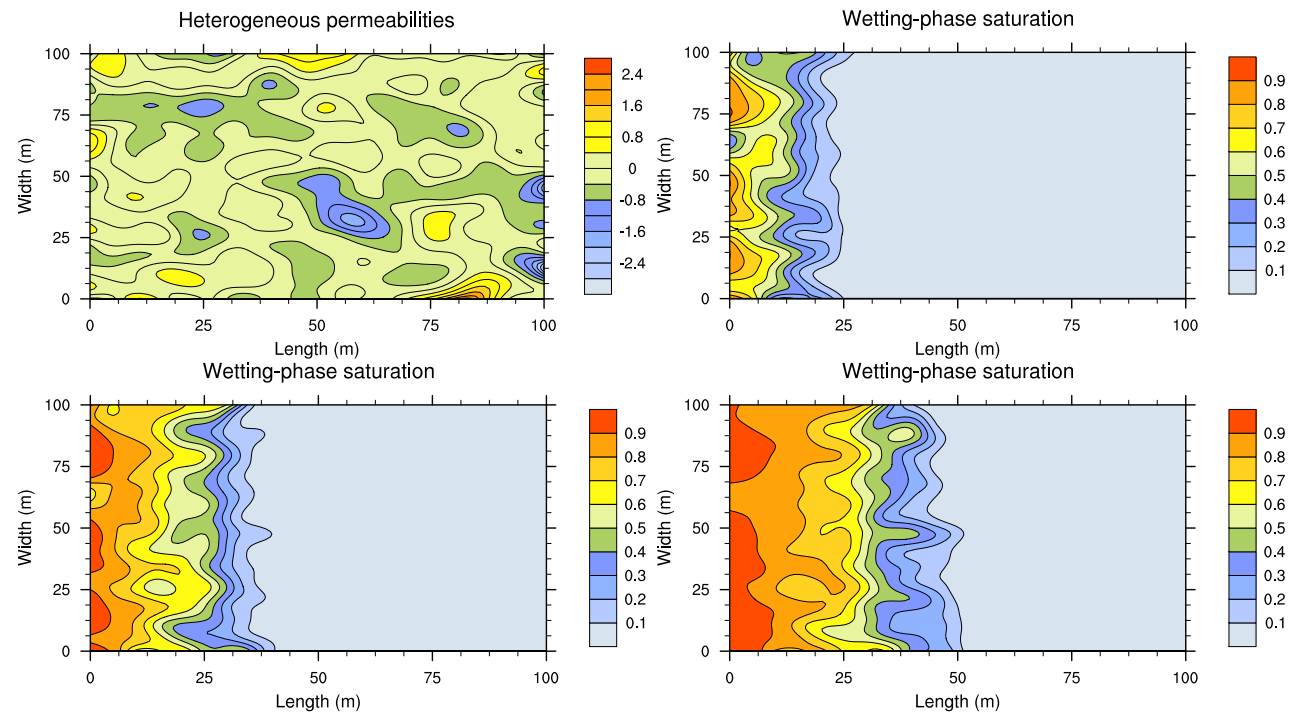

FIG. 5. Case-5: Heterogeneous permeabilities $(\log (k)$, where $k$ has a unit of $m d)$ and wettingphase saturation profiles by using the adaptive $R S-N E$ method. The mesh is $100 \times 100$. The top-left panel is for the heterogeneous permeabilities; the top-right panel is for wetting-phase saturation profiles at 0.1 PVI; the bottom-left panel is for wetting-phase saturation profiles at 0.2 PVI; the bottom-right panel is for wetting-phase saturation profiles at 0.3 PVI.

time step size $\triangle t$. We then compare in detail the classical INB and RS methods with the adaptive RS-NE method.

4.2.1. The choice of parameters in adaptive RS-NE. A threshold $N_{\text {switch }}$ is used to determine the moment of freezing the global nonlinear problems and turning to the nonlinear elimination step, in order to balance the costs and accuracy of solving the global and subspace nonlinear problems. So in this test we focus on the performance of the adaptive RS-NE method with different thresholds $N_{\text {switch }}$ for the first and second time steps, as shown in Table 4. The table presents the total computing time, the number of global nonlinear iterations, and the number of subspace nonlinear iterations and, in parentheses, the average number of iterations for solving the global and subspace Jacobian systems, respectively. The tests are done on a fixed $400 \times 200$ mesh using eight processors. We set the initial time step size to be $\Delta t=2.5 \times 10^{-3}$ for Case- 3 and $\triangle t=5 \times 10^{-3}$ for Case- 4 , respectively. It is observed from the table that (a) when $N_{\text {switch }}$ is small, such as two, the proposed adaptive RS-NE method works poorly or even fails to converge; (b) when we increase $N_{\text {switch }}$, the number of the global linear iterations becomes larger, which may degrade the performance of the RS-NE method. Based on the above observations, we find that the RS-NE method with the threshold $N_{\text {switch }}$ around five is appropriate in terms of the total computing time and the robustness and stability of the solver.

In the adaptive RS-NE method, we adaptively control the time step size $\triangle t$ by using the strategy (3.14). In the simulation, $\rho \in(0,+\infty)$ is a safeguard to avoid excessive change of the time step size between any two immediate time steps, and $\eta \in(0,1]$ is used to control the adjustment of the time step size. Hence, the parameters $\rho$ and $\eta$ are used to determine the maximum allowable time step size in the simulation. In Tables 5 and 6 , we show the effect of the parameters for Case- 3 and Case-4. It is observed from the results that the average time step size will increase 
TABLE 4

A comparison of the threshold $N_{\text {switch }}$ for Case-3 and Case-4. In the table, "Nonlinear" denotes the number of inexact nonlinear iterations per time step, "Linear" denotes the average number of GMRES iterations per nonlinear iteration, and "Time" denotes the total computing time in seconds. "Global" ("Local") denotes the performance of RS-NE in the global (subspace) iteration and "1st-step" ("2nd-step") denotes the performance of RS-NE at the first (second) time step. The “**” means divergence of the nonlinear iteration.

\begin{tabular}{|cc|ccc|ccc|}
\hline Case & \multirow{2}{*}{$N_{\text {switch }}$} & \multicolumn{2}{|c|}{ 1st-step: Global (Local) } & \multicolumn{3}{c|}{ 2nd-step: Global (Local) } \\
\cline { 3 - 8 } & & Nonlinear & Linear & Time & Nonlinear & Linear & Time \\
\hline Case-3 & 2 & & $* *$ & & & $* *$ & \\
& 5 & $15(8)$ & $30.3(1.0)$ & 29.6 & $15(12)$ & $26.0(1.6)$ & 29.1 \\
& 10 & $19(7)$ & $31.2(1.2)$ & 31.3 & $16(10)$ & $27.6(1.7)$ & 29.4 \\
& 15 & $26(6)$ & $34.7(1.3)$ & 48.6 & $18(8)$ & $27.7(1.8)$ & 34.1 \\
\hline Case-4 & 2 & $34(101)$ & $24.9(1.0)$ & 134.9 & $18(34)$ & $22.5(1.0)$ & 55.8 \\
& 5 & $14(8)$ & $41.5(1.0)$ & 36.8 & $17(18)$ & $24.1(1.0)$ & 36.9 \\
& 10 & $19(6)$ & $42.8(1.0)$ & 39.8 & $16(10)$ & $26.4(1.0)$ & 29.6 \\
& 15 & $18(2)$ & $46.7(1.0)$ & 42.7 & $17(8)$ & $28.8(1.0)$ & 32.5 \\
\hline
\end{tabular}

TABLE 5

A comparison of $\rho$ and $\eta$ for Case-3. The initial time step size is $\Delta t=0.01 .100 \times 50$ mesh.

\begin{tabular}{|l|c|c|c|c|c|}
\hline$\eta=0.75$ & $\rho=3.5$ & $\rho=3.0$ & $\rho=2.5$ & $\rho=2.0$ & $\rho=1.5$ \\
\hline Number of time steps & 30 & 35 & 42 & 52 & 68 \\
Average time step size (years) & 0.0845 & 0.0729 & 0.0610 & 0.0490 & 0.0368 \\
Average nonlinear iteration & 17.5 & 14.8 & 12.4 & 10.5 & 8.3 \\
Execution time (second) & 29.8 & 30.5 & 30.9 & 33.7 & 36.7 \\
\hline$\rho=2.5$ & $\eta=1.0$ & $\eta=0.9$ & $\eta=0.7$ & $\eta=0.6$ & $\eta=0.5$ \\
\hline Number of time steps & 31 & 35 & 44 & 50 & 56 \\
Average time step size (years) & 0.0814 & 0.0726 & 0.0574 & 0.0511 & 0.0453 \\
Average nonlinear iteration & 17.5 & 14.9 & 11.8 & 10.7 & 11.0 \\
Execution time (second) & 30.2 & 29.8 & 31.2 & 33.3 & 38.0 \\
\hline
\end{tabular}

TABLE 6

A comparison of $\rho$ and $\eta$ for Case-4. The initial time step size is $\Delta t=0.01 .200 \times 100$ mesh.

\begin{tabular}{|l|c|c|c|c|c|}
\hline$\eta=0.75$ & $\rho=3.5$ & $\rho=3.0$ & $\rho=2.5$ & $\rho=2.0$ & $\rho=1.5$ \\
\hline Number of time steps & 53 & 62 & 75 & 93 & 125 \\
Average time step size (years) & 0.1031 & 0.0883 & 0.735 & 0.587 & 0.0439 \\
Average nonlinear iteration & 14.6 & 12.4 & 10.4 & 9.2 & 7.9 \\
Execution time (second) & 433.0 & 390.4 & 397.8 & 527.9 & 522.4 \\
\hline$\rho=2.5$ & $\eta=1.0$ & $\eta=0.9$ & $\eta=0.7$ & $\eta=0.6$ & $\eta=0.5$ \\
\hline Number of time steps & 52 & 60 & 80 & 93 & 107 \\
Average time step size (years) & 0.1055 & 0.0913 & 0.0683 & 0.0592 & 0.0512 \\
Average nonlinear iteration & 15.1 & 13.3 & 9.3 & 8.9 & 7.8 \\
Execution time (second) & 370.9 & 387.6 & 397.7 & 438.6 & 465.3 \\
\hline
\end{tabular}

and fewer time steps are required when we use larger $\rho$ and $\eta$, in general resulting in the reduction of the total computing time; meanwhile it leads to the number of average nonlinear iterations decreases, hence the cost for solving nonlinear problems may increase. Observed from the tables, we find that $\rho \in(1.5,3.5)$ and $\eta \in(0.5,1]$ are appropriate to guarantee the convergence of the proposed method.

4.2.2. A comparison of INB and RS. The price to pay for using implicit methods for the fully coupled problem is to solve a nonlinear system (3.13) at each time step. When solving such nonlinear systems using the Newton method or its many variations, however, we face the numerical challenges arising from physically feasible saturation fractions between 0 and 1 . As shown in Figure 6, the INB method 

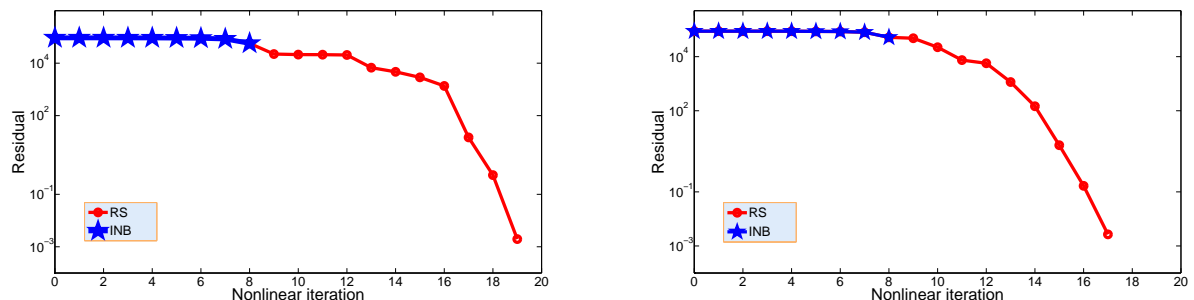

FIG. 6. Nonlinear residual history for Case-3 (left) and Case-4 (right) at the first time step using the INB and RS methods. The time step size is $\triangle t=0.01$.
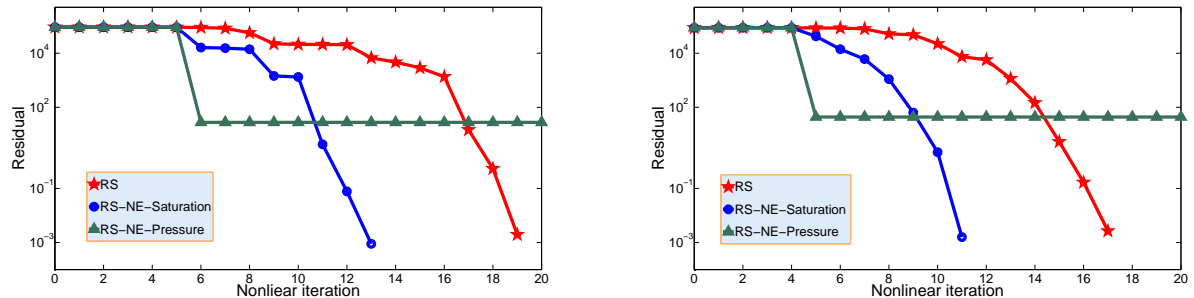

FIG. 7. Nonlinear residual history for Case-3 (left) and Case-4 (right) at the first time step. In the adaptive $R S-N E$ method, the initial time step size is $\triangle t=0.01$.

fails after eight Newton iterations, which is caused by the floating point exception from the restriction of the saturation variable. Hence, in the study we use the class of active-set reduced-space methods to fix this issue. However, along with the simulation moving onward, the nonlinear system becomes higher nonlinearity arising from heterogeneous permeability of high contrast, strong nonlinearities of relative permeability, and spatially varied capillary pressure functions. As a result, the nonlinear system is extremely difficult to solve, and the classical RS method is not convergent when it attempts to do the first nonlinear iteration. The divergence of the nonlinear iteration for the RS method is caused by the failure of linear search. Hence, we apply the NE-RS method to resolve the issue.

4.2.3. A comparison of RS and adaptive RS-NE. The RS-NE method consists of two major ingredients: a subspace correction and a global update. In the subspace correction step, the effective choice of the components to be eliminated plays an important role in the success of the whole algorithm. The RS-NE method may be not convergent when suitable components are not eliminated properly. Hence, we investigate the influence of the choices on the components to be eliminated. In the test, we carry out two different strategies: the pressure approach (i.e., removing the $p_{w^{-}}$-component) and the wetting-phase saturation approach (i.e., removing the $S_{w^{-}}$ component). In Figure 7, we show the results with the choices of removing components for Case- 3 and Case- 4 at the first time step. We can see that the pressure approach cannot make any progress after several global nonlinear iterations are finished and then we turn to the first subspace correction. This divergence of the nonlinear iteration for the pressure approach is caused by the failure of linear search. On the other hand, by using the second strategy, the RS-NE method can make a profit from the subspace correction, and the number of nonlinear iterations in the global step is smaller than that for the classical RS method. We would like to point out that the 

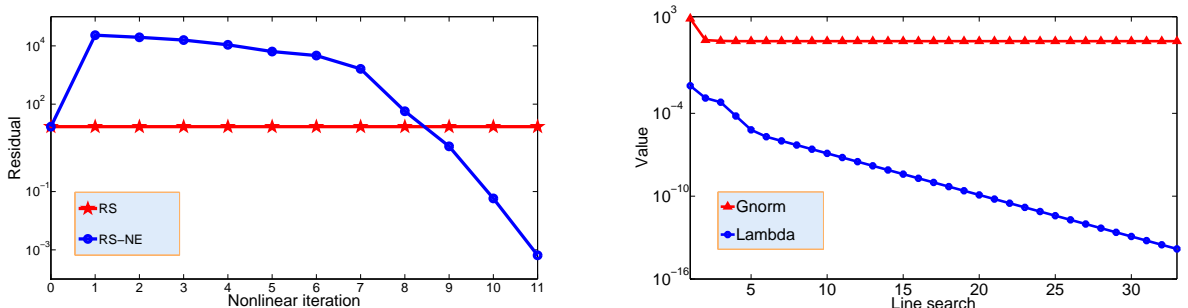

FIG. 8. Case-3: A comparison of $R S$ and adaptive RS-NE. Left panel: Nonlinear residual history at the second time step. Right panel: History of the step length $\lambda$ when it attempts to do the first nonlinear iteration. The mesh is $100 \times 50$, and the initial time step size is $\triangle t=0.01$.
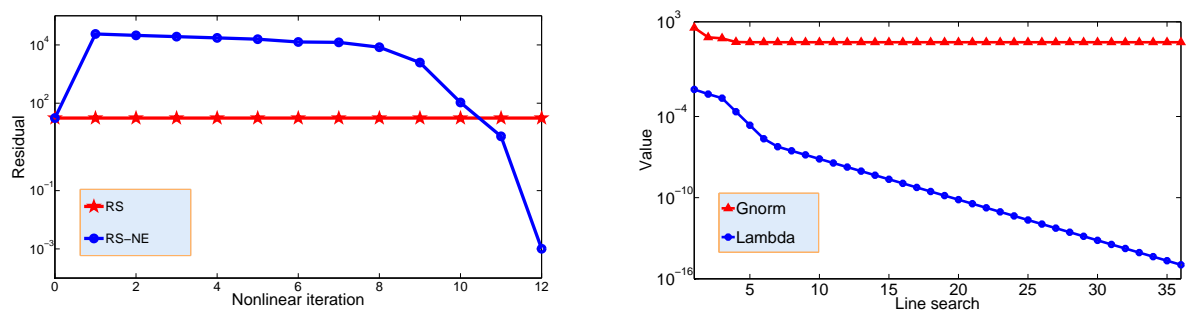

FIG. 9. Case-4: A comparison of RS and adaptive RS-NE. Left panel: Nonlinear residual history at the second time step. Right panel: History of the step length $\lambda$ when it attempts to do the first nonlinear iteration. The mesh is $200 \times 100$, and the initial time step size is $\triangle t=0.01$.

pressure approach always fails to work for two-phase flow problems at other time steps. Hence, in all the following tests we will choose the second strategy for the subspace correction.

We next investigate the performance of the adaptive RS-NE method with the classical RS method at the second time step. In Figures 8 and 9, we show the nonlinear residual history for Case-3 and Case-4, respectively. From the left panel of the figures, one can see that the nonlinear system is extremely difficult to solve, and the classical RS method is not convergent when it attempts to do the first nonlinear iteration. The divergence of the nonlinear iteration for the RS method is caused by the failure of the linear search. To figure out the reason for the failure, in the right panel of Figures 8 and 9 , we plot the histories of the step length $\lambda$ (denoted by the blue line) and the norm of nonlinear residual functions (denoted by the red line) when it attempts to do the first nonlinear iteration. In the process of the first nonlinear iteration, we first find the search direction by solving inexactly the Jacobian system, then try to compute the next approximate solution along this search direction by using a step length $\lambda$. The purpose of step length is to determine how far we should go from the current approximation and meanwhile make sure the reduction of nonlinear residual function. However, as seen in the right panel of the figures, the value of the nonlinear residual function stagnates around 10 without any improvement after dozens of line searches, even for tiny values of step length with $\lambda=10^{-15}$, while with the help of nonlinear elimination, it is clear that RS-NE converges much easier and can reach convergence with up to 12 iterations for these test cases. So we conclude that the performance of the RS-NE method is better than that of the classical RS method. We also remark that the classical RS method begins to fail to converge for any time steps $l \geqslant 2$. 

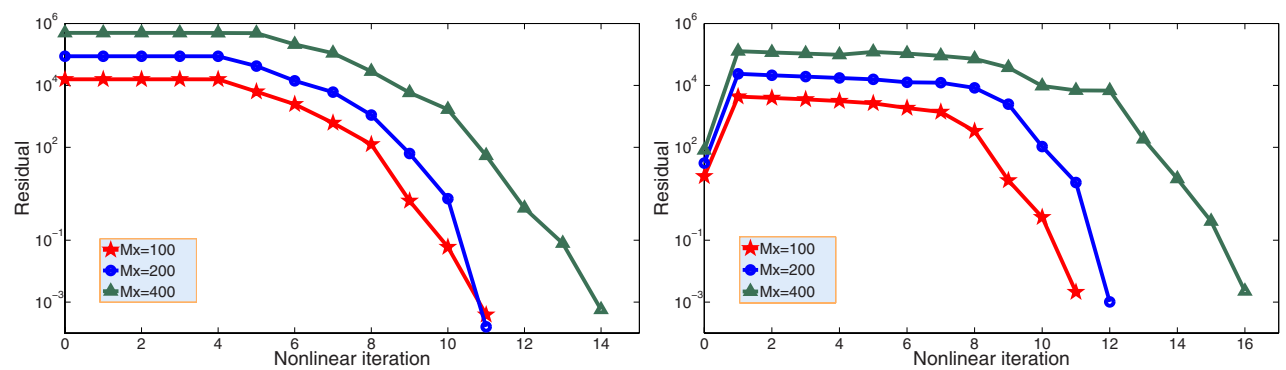

FIG. 10. Case-4: A comparison of adaptive RS-NE with different mesh sizes. Left panel: Nonlinear residual history at the first time step. Right panel: Nonlinear residual history at the second time step.

Finally, we focus on Case- 4 and study the convergence behavior when the mesh size is gradually refined from $100 \times 50$ to $400 \times 200$. In the test, the initial time step size is $\triangle t=0.02$ for the case of a $100 \times 50$ mesh and then is reduced by half as the mesh is refined accordingly. Figure 10 shows the nonlinear residual history at the first and second time steps. It is observed from the figure that as the mesh is refined the adaptive RS-NE method always converges and the number of nonlinear iterations only increases very slowly.

4.3. Parallel performance study. Scalability is an important issue in parallel computing, and the issue is more significant when solving large-scale problems with many processors. Therefore, we numerically investigate the weak and strong scalabilities of the proposed algorithm for Case- 4 in terms of the computing time and iterations.

A weak scaling test is carried out to examine the performance of the solver when the problem size is increased in proportion to the number of processor cores. In this test, we are particularly interested in stopping the calculation at a target simulation time. In the test, we start with two processor cores and a $128 \times 64$ mesh, and the number of processor cores is increased as the mesh is refined accordingly. We show in Table 7 the results obtained with the fully implicit method with adaptive time stepping and the semi-implicit method. In the adaptive fully implicit method, the initial time step size is $\Delta t=0.02$ for the case of a $128 \times 64$ mesh and then is reduced by half as the mesh is refined accordingly. In the semi-implicit method, the time step size is fixed to $\triangle t=10^{-3}$ for the case of a $128 \times 64$ mesh and then is reduced by half as the mesh is refined accordingly. However, the IMPES method is not convergent for the cases of a $512 \times 256$ mesh with $\triangle t=2.5 \times 10^{-4}$ and a $1024 \times 512$ mesh with $\triangle t=6.25 \times 10^{-5}$. Hence, we choose smaller time step sizes for these two cases. The simulation is terminated at 0.11 PVI. Table 7 gives a comparison between the computing time results of the fully implicit method with adaptive time stepping and the semi-implicit method. For the semi-implicit method, the total computing time can increase by more than one order of magnitude, as the mesh is refined, which is due to the stability restriction on the time step size. For the fully implicit method, the total numbers of time steps increase as more processor cores are used. As a result, the total numbers of nonlinear and linear iterations, as well as the total compute time, increase rapidly as the mesh is refined. It is clear that the implicit approach still outperforms the semi-implicit approach, especially for finer spatial resolutions. 
TABLE 7

Weak scaling results of the adaptive RS-NE method and the IMPES method. The "--" means that the computing time is more than 10 hours.

\begin{tabular}{|l|c|c|c|c|}
\hline Number of processors & 2 & 8 & 32 & 128 \\
Mesh size & $128 \times 64$ & $256 \times 128$ & $512 \times 256$ & $1024 \times 512$ \\
\hline Initial time step size & $2 \times 10^{-2}$ & $10^{-2}$ & $5 \times 10^{-3}$ & $2.5 \times 10^{-3}$ \\
Number of time steps (Adaptive) & 12 & 19 & 31 & 51 \\
Average time step size (years) & 0.0918 & 0.0553 & 0.0331 & 0.0196 \\
Average nonlinear iteration & 12.0 & 16.9 & 21.5 & 32.0 \\
Average linear iteration & 2.2 & 14.2 & 22.8 & 31.8 \\
Execution time (second) & 15.7 & 48.0 & 165.2 & 680.8 \\
\hline Number of time steps (IMPES) & $10^{3}$ & $2 \times 10^{3}$ & $8 \times 10^{3}$ & $3.2 \times 10^{4}$ \\
Time step size (years) & $10^{-3}$ & $5 \times 10^{-4}$ & $1.25 \times 10^{-4}$ & $3.125 \times 10^{-5}$ \\
Average linear iteration & 14.0 & 83.4 & 192.2 & -- \\
Execution time (second) & 20.3 & 217.8 & 3361.2 & -- \\
\hline
\end{tabular}

TABLE 8

Strong scalability with different number of processors $N_{p}$ for $R S-N E$.

\begin{tabular}{|ll|ccc|}
\hline Mesh & $N_{p}$ & Nonlinear & Linear & Time \\
\hline $1024 \times 512$ & 2 & 4.4 & 4.5 & 416.6 \\
& 4 & 4.4 & 30.5 & 197.9 \\
& 16 & 4.4 & 42.6 & 63.2 \\
& 64 & 4.4 & 46.0 & 18.8 \\
& 128 & 4.4 & 57.8 & 12.7 \\
\hline $2048 \times 1024$ & 32 & 6.8 & 65.0 & 388.8 \\
& 64 & 6.8 & 65.4 & 169.8 \\
& 128 & 6.8 & 81.2 & 107.4 \\
& 256 & 6.8 & 91.2 & 72.6 \\
& 512 & 6.8 & 111.8 & 50.1 \\
\hline
\end{tabular}
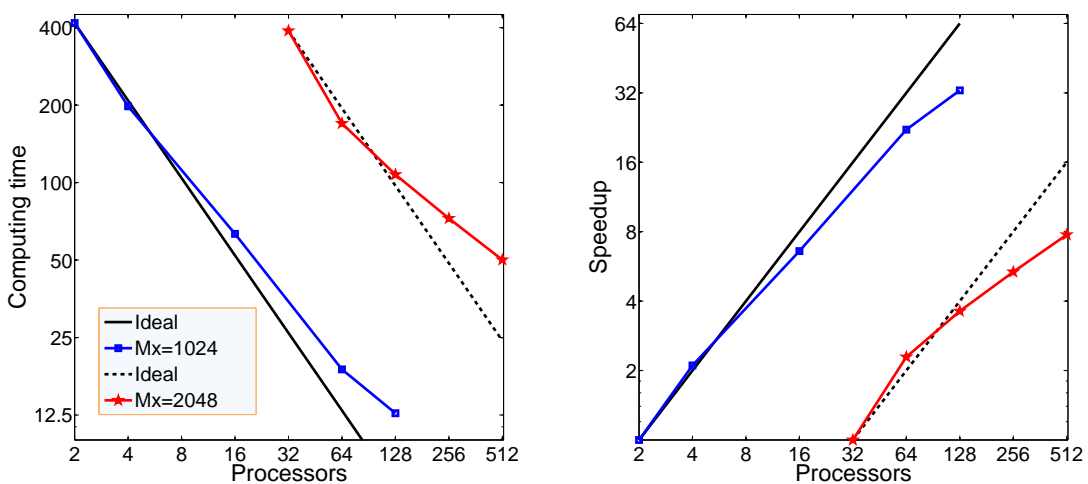

Fig. 11. Strong scalability results with different number of processors $N_{p}$ for $R S-N E$.

To further study the parallel scalability of the fully implicit method, we perform the strong scaling test on two fixed meshes and different numbers of processor cores in Table 8. The test is run with a fixed time step size $\Delta t=10^{-5}$ and the simulation is terminated at the fifth time step. We can see from the table that the number of Newton iterations does not change and the number of linear iterations increases mildly with growth of the number of processor cores. In Figure 11, we report the computing 
time with respect to the number of processors. When the number of processors $N_{p}$ increases from 2 to 512, the total computing time decreases at a reasonably good rate, which indicates that the proposed method has a good speedup for this range of numbers of processors.

5. Conclusions. In this paper, we have proposed an active-set reduced-space method with nonlinear elimination for the fully implicit simulation of two-phase flows. In the proposed algorithm, we present a variational inequality formulation of twophase flow problems to avoid nonphysical undershoot or overshoot of the saturation fractions, and we employ an active-set reduced-space algorithm to solve the resultant nonlinear complementarity system arising at each implicit time step. To improve the robustness of the nonlinear solve, a field-split nonlinear elimination preconditioner is proposed to remove the high nonlinearity that often triggers the failure of convergence. Numerical results for several test cases have shown that the proposed algorithm is robust and superior to the classical IMPES method. Although our discussion in this paper is restricted to the two-dimensional two-phase flows, we believe that the general methodology is applicable to the other nonlinear multiphase flow problems in two or three dimensions.

Acknowledgments. The authors would like to thank the anonymous reviewers for the valuable suggestions leading to the improvement of the paper.

\section{REFERENCES}

[1] S. Balay, S. Abhyankar, F. A. Adams, J. Brown, P. Brune, K. Buschelman, L. Dalcin, V. Eijkhout, W. D. Gropp, D. Kaushik, M. G. Knepley, L. C. McInnes, K. Rupp, B. F. Smith, S. Zampini, And H. Zhang, PETSc Users Manual, Argonne National Laboratory, 2015.

[2] S. J. Benson And T. S. Munson, Flexible complementarity solvers for large-scale applications, Optim. Methods Softw., 21 (2006), pp. 155-168.

[3] M. Bergounioux, K. Ito, and K. Kunisch, Primal-dual strategy for constrained optimal control problems, SIAM J. Control Optim., 37 (1999), pp. 1176-1194.

[4] L. Blank, H. Garcke, L. Sarbu, and, V. Styles, Primal-dual active set methods for AllenCahn variational inequalities with nonlocal constraints, Numer. Methods Partial Differential Equations, 29 (2013), pp. 999-1030.

[5] J. F. Blowey and C. M. Elliott, Curvature dependent phase boundary motion and parabolic double obstacle problems, in Degenerate Diffusions (Minneapolis, MN, 1991), IMA Vol. Appl. Math. 47, Springer, New York, 1993.

[6] P. Brune, M. Knepley, B. Smith, And X. Tu, Composing scalable nonlinear algebraic solvers, SIAM Rev., 57 (2015), pp. 535-565.

[7] X.-C. Cai, W. D. Gropp, D. E. Keyes, R. G. Melvin, and D. P. Young, Parallel NewtonKrylov-Schwarz algorithms for the transonic full potential equation, SIAM J. Sci. Comput., 19 (1998), pp. 246-265.

[8] X.-C. CAi AND M. SARKIS, A restricted additive Schwarz preconditioner for general sparse linear systems, SIAM J. Sci. Comput., 21 (1999), pp. 792-797.

[9] X.-C. Cai and D. E. KeYes, Nonlinearly preconditioned inexact Newton algorithms, SIAM J. Sci. Comput., 24 (2002), pp. 183-200.

[10] X.-C. CAI AND X. LI, Inexact Newton methods with restricted additive Schwarz based nonlinear elimination for problems with high local nonlinearity, SIAM J. Sci. Comput., 33 (2011), pp. $746-762$.

[11] Z. Chen, G. Huan, And B. Li, An improved IMPES method for two-phase flow in porous media, Transport Porous Media, 54 (2004), pp. 361-76.

[12] Z. Chen, G. Huan, And Y. Ma, Computational Methods for Multiphase Flows in Porous Media, SIAM, Philadelphia, PA, 2006.

[13] K. H. CoATs, IMPES stability: the CFL limit, presented at SPE Reservoir Simulation Symposium, Houston, TX, 2001.

[14] C. N. Dawson, H. Klie, M. F. Wheeler, and C. S. Woodward, A parallel, implicit, cellcentered method for two-phase flow with a Newton-Krylov solver, Comput. Geosci., 1 (1997), pp. 215-249. 
[15] J. E. Dennis And R. B. Schnabel, Numerical Methods for Unconstrained Optimization and Nonlinear Equations, SIAM, Philadelphia, 1996.

[16] V. Dolean, M. J. Gander, W. Kherij, F. Kwok, and R. Massin, Nonlinear Preconditioning: How to Use a Nonlinear schwarz to Precondition Newton's Method, preprint, 2015.

[17] S. C. Eisenstat And H. F. Walker, Globally convergent inexact Newton method, SIAM J. Optim., 4 (1994), pp. 393-422.

[18] I. Ekeland and R TÉmam, Convex Analysis and Variational Problems, SIAM, Philadelphia, 1999.

[19] R. G. Fagin and C. H. Stewart, JR., A new approach to the two-dimensional multiphase reservoir simulator, SPE J., 6 (1966), pp. 175-182.

[20] R. Glowinski, Numerical Methods for Nonlinear Variational Problems, Springer-Verlag, Berlin, 2008.

[21] P. T. HARKer And J. S. PANG, Finite-dimensional variational inequality and nonlinear complementarity problems: A survey of theory, algorithms and applications, Math. Program., 48 (1990), pp. 161-220.

[22] M. HintermüLleR, K. Ito, AND K. Kunisch, The primal-dual active set strategy as a semismooth Newton method, SIAM J. Optim., 13 (2003), pp. 865-888.

[23] M. Hintermüller, V. A. Kovtunenko, And K. Kunisch, The primal-dual active set method for a crack problem with non-penetration, IMA J. Appl. Math., 69 (2004), pp. 1-26.

[24] H. Hoteit And A. Firoozabadi, Numerical modeling of two-phase flow in heterogeneous permeable media with different capillarity pressures, Adv. Water Resources, 31 (2008), pp. $56-73$.

[25] F.-N. Hwang AND X.-C. CAI, A parallel nonlinear additive Schwarz preconditioned inexact Newton algorithm for incompressible Navier-Stokes equations, J. Comput. Phys., 204 (2005), pp. 666-691.

[26] F.-N. Hwang, H.-L. Lin, AND X.-C. CAI, Two-level nonlinear elimination based preconditioners for inexact Newton methods with application in shocked duct flow calculation, Electron. Trans. Numer. Anal., 37 (2010), pp. 239-251.

[27] F.-N. Hwang, Y.-C. SU, AND X.-C. CAI, A parallel adaptive nonlinear elimination preconditioned inexact Newton method for transonic full potential flow problems, Comput. \& Fluids, (2015), pp. 96-107.

[28] K. ITO AND K. Kunisch, The primal-dual active set method for nonlinear optimal control problems with bilateral constraints, SIAM J. Control Optim., 43 (2004), pp. 357-376.

[29] P. J. Lanzkron, D. J. Rose, And J. T. Wilkes, An analysis of approximate nonlinear elimination, SIAM, J. Sci. Comput., 17 (1996), pp. 538-559.

[30] A. Klawonn, M. Lanser, and O. Rheinbach, Nonlinear FETI-DP and BDDC Methods, SIAM, J. Sci. Comput., 36 (2014), pp. A737-A765.

[31] D. A. Knoll and D. E. Keyes, Jacobian-free Newton-Krylov methods: a survey of approaches and applications, J. Comput. Phys., 193 (2004), pp. 357-397.

[32] J. Kou AND S. Sun, A new treatment of capillarity to improve the stability of IMPES two-phase flow formulation, Comput. \& Fluids, 39 (2010), pp. 1293-1931.

[33] J. Kou And S. Sun, Convergence of discontinuous Galerkin methods for incompressible twophase flow in heterogeneous media, SIAM J. Numer. Anal., 51 (2013), pp. 3280-3306.

[34] D. Krishnan, P. Lin, AND A. M. YIP, A primal-dual active-set method for non-negativity constrained total variation deblurring problems, IEEE Trans. Image Process., 16 (2007), pp. 2766-2777.

[35] D. KRishnan, Q. Pham, AND A. M. YiP, A primal-dual active-set algorithm for bilaterally constrained total variation deblurring and piecewise constant Mumford-Shah segmentation problems, Adv. Comput. Math., 31 (2009), pp. 237-266.

[36] F. KwoK AND H. TChelePI, Potential-based reduced newton algorithm for nonlinear multiphase flow in porous media, J. Comput. Phys., 227 (2007), pp. 706-727.

[37] L. W. Lake, Enhanced Oil Recovery, Prentice-Hall, Englewood Cliffs, NJ, 1989.

[38] K. Lie, S. Krogstad, I. Ligaarden, J. Natvig, H. Nilsen, and B. Skaflestad, Open-source MATLAB implementation of consistent discretisations on complex grids, Comput. Geosci., 16 (2012), pp. 297-322.

[39] L. LiU And D. E. Keyes, Field-split preconditioned inexact Newton algorithms, SIAM J. Sci. Comput., 37 (2015), pp. A1388-A1409.

[40] D. Loudyi, A 2D Finite Volume Model for Groundwater Flow Simulations: Integrating Nonorthogonal Grid Capability into MODFLOW, Ph.D. dissertation, Cardiff University, UK, 2005.

[41] J. E. P. Monteagudo and A. Firoozabadi, Control-volume method for numerical simulation of two-phase immiscible flow in two- and three-dimensional discrete-fracture media, Water Resources Res., 40 (2004), W07405.

Copyright $@$ by SIAM. Unauthorized reproduction of this article is prohibited. 
[42] J. E. P. Monteagudo And A. Firoozabadi, Comparison of fully implicit and IMPES formulations for simulation of water injection in fractured and unfractured media, Int. J. Numer. Methods Engrg., 69 (2007), pp. 698-728.

[43] J. Moortgat, S. Sun, And A. Firoozabadi, Compositional modeling of three-phase flow with gravity using higher-order finite element methods, Water Resources Res., 47 (2011), pp. W05511.

[44] W. A. Mulder And B. Van Leer, Experiments with implicit upwind methods for the Euler equations, J. Comput. Phys., 59 (1985), pp. 232-246.

[45] T. S. Munson, F. Facchinei, M. C. Ferris, A. Fischer, and C. Kanzow, The semi-smooth algorithm for large scale complementarity problems, INFORMS J. Comput., 13 (2001), pp. 294-311.

[46] M. Munteanu, L. F. Pavarino, and S. SCACChi, A scalable Newton-Krylov-Schwarz method for the bidomain reaction-diffusion system, SIAM J. Sci. Comput., 31 (2009), pp. 3861-3883.

[47] M. Osusky and D. W. ZingG, Parallel Newton-Krylov-Schur flow solver for the Navier-Stokes equations, AIAA J., 51 (2013), pp. 2833-2851.

[48] J. Pebrel, C. Rey, and P. Gosselet, A nonlinear dual-domain decomposition method: Application to structural problems with damage, Internat. J. Multiscale Comput. Engrg., 6 (2008), pp. 251-262.

[49] J. F. Rodrigues, Obstacle Problems in Mathematical Physics, North-Holland, Amsterdam, 1987.

[50] Y. SAAD, Iterative Methods for Sparse Linear Systems, 2nd ed., SIAM, Philadelphia, 2003.

[51] J. O. Skogestad, E. Keilegavlen, and J. M. Nordbotten, Domain decomposition strategies for nonlinear flow problems in porous media, J. Comput. Phys., 234 (2013), pp. 439-451.

[52] B. Smith, P. BJørstad, And W. Gropp, Domain Decomposition: Parallel Multilevel Methods for Elliptic Partial Differential Equations, Cambridge University Press, Cambridge, UK, 1996.

[53] S. Sun, D. E. KeYes, And L. Liu, Fully implicit two-phase reservoir simulation with the additive Schwarz preconditioned inexact Newton method, presented at SPE Reservoir Characterization and Simulation Conference and Exhibition, Society of Petroleum Engineers, 2013.

[54] P. Sun, G. Xue, C. WANG, And J. Xu, Fast numerical simulation of two-phase transport model in the cathode of a polymer electrolyte fuel cell, Commun. Comput. Phys., 6 (2009), pp. $49-71$.

[55] A. Toselli And O. Widlund, Domain Decomposition Methods-Algorithms and Theory, Springer-Verlag, Berlin, 2005.

[56] P. Wilmott, J. Dewynne, And S. Howison, Option Pricing, Oxford, England, Oxford Financial Press, Oxford, UK, 1993.

[57] Y. Wu AND G. QIN, A generalized numerical approach for modeling multiphase flow and transport in fractured porous media, Commun. Comput. Phys., 6 (2009), pp. 85-108.

[58] C. YANG AND X.-C. CAI, A scalable fully implicit compressible Euler solver for mesoscale nonhydrostatic simulation of atmospheric flows, SIAM J. Sci. Comput., 35 (2014), pp. S23-S47.

[59] L. C. Young And R. E. Stephenson, A generalized compositional approach for reservoir simulation, SPE J., 23 (1983), pp. 727-742.

Copyright $@$ by SIAM. Unauthorized reproduction of this article is prohibited. 